\title{
L'analyse de variation monotone pour les moyennes et les proportions
}

\author{
Louis Laurencelle ${ }^{\mathrm{a} \otimes}$ \\ ${ }^{a}$ Université du Québec à Trois-Rivières
}

\begin{abstract}
Entre la variation statistique au hasard et la variation linéaire, toutes deux bien connues, il existe d'autres modèles de variation (polynomiale linéaire, périodique, etc.) et, notamment, la variation monotone. D'une condition à l'autre dans une série à contrôle croissant, d'un niveau d'intervention ou d'une dose médicinale à l'autre, la variable mesurée reflète-t-elle un effet consistant, croissant ou décroissant, cela sans qu'un modèle précis puisse lui être sous-tendu ? L'analyse de variation monotone, intégrée à l'analyse de variance classique, permet de décider si la variable observée répond de façon cohérente à une variable indépendante en escalier, celle-ci étant de type ordinal plutôt que linéaire. Nous présentons deux techniques adaptées à l'analyse de variance, celle de Barlow et collaborateurs (1972) et celle d'Abelson et Tukey (2013), permettant de rejeter l'hypothèse nulle en regard d'une hypothèse d'évolution consistance de la variable observée, l'application étant étendue à l'analyse des proportions. Des exemples, des tables de valeurs critiques et de coefficients et les formules de base sont aussi fournis. $\square$ Between random statistical variation and simple linear change, both of which are well known, there are other models of variation (linear polynomials, periodic functions, etc.) and, particularly, that of monotonic variation. From one condition to the next in an increasing control series, from one intervention level or medicinal dose to the next, does the measured variable reflect a consistent, increasing or decreasing effect, without a definite mathematical pattern underlying it? Monotonic variation analysis, integrated with classical ANOVA, is used to decide whether the observed variable responds consistently to a staircase independent variable, which is ordinal rather than linear. We present here two techniques for ANOVA, that of Barlow and colleagues (1972) and that of Abelson and Tukey (2013), allowing the rejection of the null hypothesis against a hypothesis of consistent evolution of the observed variable; the application is extended to the analysis of proportions. Examples, tables of critical values and coefficients, and basic formulas are also provided.
\end{abstract}

Keywords $₫$ Variation monotone, Analyse de variance, Analyse de proportions $\square$ Monotonic variation, Analysis of variance, Analysis of proportions.

Acting Editor $\square$ Denis Cousineau (Université d’Ottawa)

Les contenus de cet article sont partiellement empruntés à Laurencelle (1993, 2014) louis.laurencelle@gmail.com

10.20982/tqmp.17.4.p374

\section{Introduction}

La comparaison de moyennes en analyse de variance a pour but de vérifier si les différences observées entre elles sont attribuables à un facteur expérimental ou classificatoire ou si on peut les réduire à de seules fluctuations aléatoires. La procédure de test permet de décider entre une interprétation invoquant le hasard seul ou celle d'une variation systématique, causée supposément par la variable indépendante.
Or, pour l'analyse de variance, il existe différents types de variables indépendantes et les tests appropriés ne sont pas toujours les mêmes pour l'une et l'autre. Les $k$ niveaux de la variable indépendante, ou $k$ variantes, peuvent dénoter des quantités, des intensités ou des catégories. La plupart des tests connus, tels la procédure HSD de Tukey, les tests de Dunett, Newman-Keuls ou Scheffé (voir Winer, Brown et Michels, 1991), concernent principalement les variables catégorielles, ou exploitées comme telles. Pour les quantités, c'est-à-dire les variables consti- 
tuant une métrique, l'analyse en polynômes linéaires orthogonaux (ibid.) ou l'analyse de régression (Draper et Smith, 1981) s'appliquent. Il arrive toutefois qu'on exploite une variable indépendante située à mi-chemin entre ces deux pôles, une variable semi-métrique ou ordinale, représentant seulement une relation d'ordre entre elle et la variable dépendante. Pour les $k=4$ valeurs $v_{1}, v_{2}, v_{3}$ et $v_{4}$, nous pourrions par exemple stipuler la relation d'ordre :

$$
v_{1} \leq\left\{v_{2}, v_{3}\right\} \leq v_{4} \text {. }
$$

Cette relation stipule trois groupes de valeurs croissantes, ou plus exactement non décroissantes, qui correspondent aux conditions d'expérience $c_{1}, c_{2}, c_{3}$ et $c_{4}$, les éléments $v_{2}$ et $v_{3}$ du second groupe n'étant imposés d'aucune relation mutuelle.

L'expérimentation d'un nouveau produit, un régime alimentaire, un programme d'entraînement, une intervention médicale (vaccins, médicaments, drogues), est l'illustration classique d'une relation d'ordre. Par exemple, la condition de contrôle, celle évaluée sans intervention, est comparée à $k-1$ conditions à niveaux croissants de l'intervention : quantité de protéines quotidiennes, dose du vaccin, heures d'entraînement quotidien, le tout représenté par la quasi inégalité suivante :

$$
v_{1} \leq v_{2} \leq \cdots \leq v_{k}
$$

Enfin, l'utilisation d'une variable indépendante métrique peut aussi commander une relation d'ordre hors modèle si la fonction causale $f$, dans :

(variable dépendante $Y)=f($ variable indépendante $X)$,

est elle-même élastique ou incomplètement spécifiée. Ainsi, si l'on mesure la perception rapide de mots de deux syllabes $(Y)$ en fonction du niveau d'éclairage ou de luminosité $(X)$, on pourra formuler l'hypothèse d'ordre suivante :

$$
H_{1}: Y_{i} \geq Y_{j} \text { si } Y=f_{\text {monotone }}(X) \text { et } X_{i} \geq X_{j} \text {. }
$$

Comme le lecteur l'aura compris, la fonction invoquée,

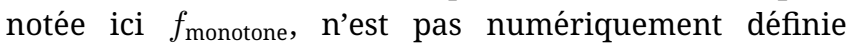
comme l'est une fonction mathématiquement structurée telle que la fonction linéaire simple, $Y=b_{0}+b_{1} \cdot X$. Elle spécifie seulement que, pour toute variation de $X$, la variable indépendante $Y$ ne variera que dans un seul sens. Pour un nouvel exemple, si l'hypothèse d'ordre stipule que les niveaux croissants de $X$ feront diminuer la variable dépendante $Y$, alors les valeurs successives de $Y$ ne devraient jamais croître, l'hypothèse se formulant alors par :

$$
H_{1}: Y_{i} \leq Y_{j} \text { si } Y=f_{\text {monotone }}(X) \text { et } X_{i} \geq X_{j}
$$

ou, plus simplement, $H_{1}: Y_{1} \leq Y_{2} \leq \cdots \leq Y_{k}$. Nombre de cas pareils se rencontrent en recherche pour lesquels les procédures appliquées manquent de puissance parce qu'elles n'exploitent pas explicitement l'hypothèse globale proposée. Par exemple, une pratique encore répandue consiste à comparer les $k$ moyennes observées par paires de niveaux successifs. Quant au pis-aller de l'analyse polynomiale linéaire, une procédure globale celle-ci, l'hypothèse et la métrique qu'elle exploite dépassent le modèle expérimental stipulé.

Il existe quelques approches pour établir la significativité statistique d'une variation monotone de la variable dépendante. Notons d'emblée que, sous réserve de l'erreur de mesure, la probabilité que $k$ moyennes se retrouvent par hasard dans un ordre monotone strict, par exemple $\bar{X}_{1}<\bar{X}_{2}<\ldots<\bar{X}_{k}$, est de $1 / k$ !. Pour $k=4$ niveaux, l'obtention d'un tel ordre satisferait le seuil de significativité de 0,05 , sa probabilité étant de $1 / 4 !=1 / 24 \approx 0,042$ : pourquoi aller plus loin? Jonckeere (1954) propose un meilleur test non-paramétrique, Hayter (1990) encore un autre en forme de test $t$. Le test à la fois le plus spécifique et le plus puissant fut formulé par Bartholomew (1959a, 1959b, 1961; voir aussi Barlow, Bartholomew, Bremner et Brunk 1972), ce dans le contexte de l'analyse de variance, utilisant des variables aléatoires normales.

Nous présentons ici deux tests que nous jugeons plus intéressants pour décider de l'hypothèse d'une variation monotone de la variable dépendante. Ce sont deux tests globaux, celui plus rigoureux présenté dans Barlow et coll. (1972) et un test approximatif, plus simple, proposé par Abelson et Tukey (2013), ces deux tests convenant au contexte de l'analyse de variance. Deux exemples permettront d'illustrer les deux techniques, l'un plus classique portant sur une série de moyennes indépendantes, l'autre sur une série de proportions. L’Appendice enfin encapsulera la théorie mathématique du test de Bartholomew, Barlow et coll. (ibid.) et offrira notamment quelques tables de valeurs critiques (tirées de Laurencelle, 2002) et de coefficients.

Note sur les valeurs calculées. Les valeurs calculées rapportées ici sont d'une précision plus grande que n'en indiquent les décimales affichées, de sorte que, par exemple, la valeur affichée du quotient de deux valeurs affichées précédentes peut ne pas correspondre exactement. Le lecteur peut être assuré que les décimales affichées de chaque valeur rapportée sont correctes.

\section{Les tests $\bar{E}^{2}$ et $\bar{\chi}^{2}$ de Barlow et coll. (1972)}

Prenons un modèle d'analyse de variance simple, à une dimension. Dans ce modèle, nous avons $k$ groupes de $n_{j}$ observations indépendantes chacun, pour un nombre total $N=\sum_{j=1}^{k} n_{j}$ d'observations. Les moyennes des $k$ groupes 
sont $\bar{X}_{1}, \bar{X}_{2}, \ldots, \bar{X}_{k}$. Sous l'hypothèse d'homogénéité des variances, les variances dans chaque groupe sont chacune un estimé d'une variance paramétrique parente, $\sigma_{\epsilon}^{2}$, tout l'est comme l'estimé global, le Carré Moyen d'erreur (CME). Ce dernier est obtenu selon :

$$
C M E=\sum\left(n_{j}-1\right) \cdot s_{j}^{2} /(N-k),
$$

et il est doté de $\sum_{j=1}^{k}\left(n_{j}-1\right)=N-k$ degrés de liberté.

Pour tester l'hypothèse globale d'une variation systématique des données de l'ensemble considéré, nous construisons un estimé de la variance entre les moyennes, le Carré Moyen inter-groupes (CMG). Une formule possible est :

$$
C M G=\tilde{n} \cdot s^{2}\left(\bar{X}_{j}\right),
$$

où $\tilde{n}$ dénote la moyenne harmonique des tailles $n_{j}$, soit $\tilde{n}=k / \sum_{j=1}^{k} n_{j}^{-1}$ (Laurencelle, 2017); dans le cas spécial de groupes à tailles $n$ égales, $\tilde{n}=n$. Le CMG, basé sur $k$ moyennes, a $k-1$ degrés de liberté, et le quotient CMG / CME, indicateur potentiel d'un effet ajouté de variation systématique, se distribue théoriquement comme la variable $F_{k-1, N-k}$ sous la double condition d'une normalité approximative de la variable $X$ et de variances intragroupe de sources comparables. La décision contre une pure variation aléatoire des moyennes et en faveur d'une variation systématique tient à ce que le quotient obtenu déborde $F_{k-1, N-k, 1-\alpha}$, le centile $100(1-\alpha)$ de la distribution $F$.

Pour tester l'hypothèse d'une relation de variation monotone des moyennes en fonction des $k$ niveaux de la variable indépendante, il faut d'abord vérifier et, au besoin, imposer cette relation aux moyennes considérées. Cette imposition se fait par amalgamation (selon le vocable suggéré par Barlow et coll., 1972), c.-à-d. par égalisation des moyennes violatrices. Le procédé consiste à :

(a) repérer la ou les moyennes violatrices de la relation d'ordre; si la relation est déjà respectée, l'amalgamation est inutile, sinon, passer à (b);

(b) associer une moyenne violatrice à une ou plusieurs voisines, à sa gauche ou à sa droite, et remplacer chacune par leur moyenne, puis calculer la variance de la série obtenue;

(c) continuer l'aplatissement des écarts à l'ordre monotone jusqu'à satisfaction complète de la relation d'ordre et, si deux ou plusieurs séries 'amalgamées' en résultent, retenir celle qui présente la variance la plus élevée.

Prenons d'abord l'hypothèse d'ordre simple $a \leq b \leq$ $c \leq d$ et les 4 moyennes consécutives 26,$2 ; 33,5 ; 42,4$; 42,4 . Ici, la variation monotone est respectée, aucune valeur ne variant en direction contraire à celle spécifiée. Par contre, dans la série suivante, $\{12,0 ; 16,0 ; 14,0 ; 19,0\}$, la seconde valeur, 16,0, contrarie la relation spécifiée : il faut donc intervenir. Une solution serait d'" amalgamer ", c.-à-d. combiner les deux premières valeurs, produisant la série 14,$0 ; 14,0 ; 14,0 ; 19,0$, de variance 6,25 . Une autre combinaison, celle des deuxième et troisième valeurs, donnera 12,$0 ; 15,0 ; 15,0 ; 19$, de variance 8,25 , plus forte que la précédente. Le lecteur peut vérifier que cette dernière série donne la solution optimale, celle qui, à partir de la variance de 8,917 de la série originale, a conservé la plus grande portion : cette variance 'optimale' peut être désignée ${ }^{1} s^{2}\left(\bar{X}_{j}^{*}\right)$. C'est à cette série 'amalgamée' optimale que s'adresse le test de Barlow et coll. (1972).

Désignant le vecteur original de moyennes comme $\left\{\bar{X}_{1}, \bar{X}_{2}, \ldots, \bar{X}_{k}\right\}$, le procédé d'amalgamation produira un nouveau vecteur, $\left\{\bar{X}_{1}^{*}, \bar{X}_{2}^{*}, \ldots, \bar{X}_{k}^{*}\right\}_{r}$ ou, plus simplement, $\left\{\bar{X}_{1}, \bar{X}_{2}, \ldots, \bar{X}_{k}\right\}_{r}^{*}$ où le symbole $r$ dénote le nombre de valeurs algébriquement distinctes du vecteur après amalgamation. Dans notre exemple des $k=$ 4 moyennes ci-dessus, deux moyennes ont dû être combinées, laissant $r=3$ valeurs distinctes dans le vecteur à variance $s^{2}\left(\bar{X}_{j}^{*}\right)=8,25$.

Le test de variation monotone, dénoté $\bar{E}^{2}$ est un quotient de sommes de différences carrées plutôt que de Carrés Moyens comme en analyse de variance ${ }^{2}$, un cousin du coefficient de corrélation non-linéaire $\eta^{2}$. Sa forme globale est :

$$
\bar{E}^{2}=\frac{\text { somme des carrés associée aux moyennes après amalgamation }}{\text { somme des carrées totale }} .
$$

1. La variance réelle serait plutôt $s^{2}\left(\bar{X}^{*}\right) \cdot(k-1) /(r-1), r$ chiffrant le nombre de valeurs distinctes, non amalgamées, de la série et constituant aussi les degrés de liberté de la variance produite. Pour l'exemple donné un peu plus bas, de variance 'classique' (par commodité) $=8,25$, avec $k=4$ et $r=3$, la variance réelle des moyennes de la série serait de $8,25 \times 4 / 3=11$, dotée de $r-1=2$ degrés liberté.

2. Ce quotient de sommes de carrés, la " somme de carrés " représentant le numérateur de la variance échantillonnale classique, était la forme initiale que R. A. Fisher (1890-1962) avait donnée au test d’analyse de variance, sa création, ce test probabiliste référant alors à la loi Bêta. La transformation vers le quotient de variances employé aujourd’hui et la loi $F$ correspondante est due à George Snedecor (1881-1974). 
La " somme de carrés totale " est ici l'addition des 'carrés' entre groupes et des carrés intra groupes, soit $(k-1)$. $C M G+(N-k) \cdot C M E$. Quant à la « somme des carrés associée aux moyennes après amalgamation ", on l'obtient $\operatorname{par}(r-1) \cdot C M G^{*}$, où $\left.C M G^{*}=\tilde{n} \cdot s^{2}(\overline{(X})_{j}^{*}\right) \cdot(k-1) /(r-$ $1)$. Le quotient permettant d'effectuer le test est donc :

$$
\bar{E}^{2}=\frac{(r-1) \cdot C M G^{*}}{(k-1) \cdot C M G+(N-k) \cdot C M E}
$$

ou

$$
\bar{E}^{2}=\frac{(k-1) \cdot \tilde{n} \cdot s^{2}\left(\bar{X}_{j}^{*}\right)}{(k-1) \cdot \tilde{n} \cdot s^{2}\left(\bar{X}_{j}\right)+(N-k) \cdot C M E},
$$

la formule (3b) permettant d'exploiter la formule classique de variance pour les moyennes.

À l'instar du $F$ appliqué traditionnellement dans les tests de l'analyse de variance, la statistique $\bar{E}^{2}$ a deux paramètres, $k$ le nombre de groupes et $N-k$ les degrés de liberté du $C M E$ ou variance d'erreur considérée, laquelle estime la grandeur de la variation aléatoire présente.

Dans le cas de groupes de taille très grande $(n \rightarrow \infty)$ ou 'infinie' ou si la variance aléatoire paramétrique $\sigma_{\epsilon}^{2}$ est connue, le test $\bar{E}^{2}$ se traduit dans la forme du $\chi^{2}$, soit :

$$
\bar{\chi}^{2}=\frac{(r-1) \cdot C M G^{*}}{\sigma_{\epsilon}^{2}},
$$

une statistique gouvernée ici par le seul paramètre $k$, le nombre de groupes.

Ces deux statistiques, $\bar{E}^{2}$ et $\bar{\chi}^{2}$, qui reflètent l'écart à la contrainte d'une variation monotone des $k$ moyennes, ont leurs distributions propres. L'Appendice présente des tables de valeurs critiques de ces deux statistiques (reproduites de Laurencelle, 1993, p. 84-87, et 2002, p. 81-84).

\section{Le test d'Abelson et Tukey (2013)}

Plutôt qu'une approche de combinatoire rigoureuse basant les statistiques $\bar{E}^{2}$ et $\bar{\chi}^{2}$, Abelson et Tukey (2013) adoptent une approche 'géométrique' qui leur permet de proposer un test global très simple de la variation monotone, test approximatif calqué sur l'analyse de variance en composantes polynomiales orthogonales attribuée à Fisher. Il s'agit d'obtenir un carré moyen $(C M C)$ à partir d'un contraste linéaire $C$, c.-à-d. un composé linéaire pondéré des $k$ moyennes dont les coefficients somment à zéro, soit le contraste :

$$
C=c_{1} \bar{X}_{1}+c_{2} \bar{X}_{2}+\ldots+c_{k} \bar{X}_{k} \text {, tel que } \sum_{j=1}^{k} c_{j}=0
$$

et le carré moyen est :

$$
C M C=\tilde{n} \cdot C^{2} / \sum_{j-1}^{k} c_{j}^{2}
$$

$\tilde{n}$ étant la taille (moyenne) groupes. Les coefficients proposés sur une base 'géométrique' par les auteurs sont :

$$
c_{j}=\sqrt{(j-1)[1-(j-1) / k]}-\sqrt{j \cdot(1-j / k)},
$$

pour $j=1$ à $k$. Le lecteur trouvera au tableau A5, en appendice, une table des coefficients tels que définis ci-dessus pour un nombre $k$ de moyennes de 4 à 25. Le carré moyen (ou variance) associé à un contraste possède par définition 1 degré de liberté.

Par souci de commodité pour l'utilisateur, les auteurs proposent aussi de remplacer les coefficients $c_{j}$ définis cidessus par d'autres, de calcul facile : c'est leur solution « 4-2 ». Elle consiste à établir d'abord les coefficients $c_{j}$ du polynôme orthogonal de degré 1, qu'on trouve facilement, et d'en accentuer les valeurs extrêmes, soit multiplier par 4 les coefficients extrêmes $c_{1}$ et $c_{k}$ et par 2 les coefficients voisins $c_{2}$ et $c_{k-1}$. Une méthode très simple pour définir ces coefficients est d'abord d'énumérer les nombres 1 à $k$, de soustraire de chacun la valeur $(k+1) / 2$ et enfin d'appliquer l'accentuation indiquée. Le tableau 1 illustre la recette de production pour le cas $k=7$ niveaux. La série correspondante de $k$ coefficients obtenus avec (6) est $\{-0,926,-0,269,-0,114,0,00,0,114,0,269,0,926\}$, qui corrèle à 0,999 avec la série 'simple' obtenue à l'étape 3 , au tableau 1. Après standardisation des valeurs de chaque série par son écart-type, l'écart maximal entre les coefficients standardisés seulement de 0,067. Ces indices de corrélation et d'écart maximal justifient une confiance suffisante dans la solution facile pour de courtes $(k)$ séries. Dans le cas de séries plus longues $(k>50)$, l'effet des accentuations des coefficients extrêmes s'exagère. ${ }^{3}$ Que le calcul se fasse avec l'une ou l'autre des séries de coefficients, le test à faire utilise un quotient $F=C M C / C M E$, référé à la loi $F$ dotée de 1 et $N-k$ degrés de liberté dans le contexte d'une analyse de variance simple.

\section{Un premier exemple d'analyse portant sur des moyennes}

Supposons que, pour vérifier l'effet du café sur l'attention, nous formons $k=5$ groupes de 10 participants chacun, tous devant effectuer une tâche expérimentale requérant un bon contrôle visuomoteur. Avant de débuter la tâche, chaque participant absorbe du café fort : ceux du groupe 1 en boivent $40 \mathrm{ml}$, ceux du groupe $280 \mathrm{ml}$, ainsi de

3. Dans le cas de séries plus longues $(k>50)$, l'effet des accentuations des coefficients extrêmes devient suspect en ce qu'il peut imposer artificiellement l'effet « variation monotone " à une série par ailleurs aléatoire. . Par exemple, pour $k=200$, la corrélation de 0,926 permet un écart maximal de 1,820 pour les coefficients $c_{1}$ et $c_{200}$ entre la solution 'géométrique' et son approximation; ces indices sont de 0,845 et 4,752 pour $k=500$. Cet écart, si trop élevé, est susceptible de compromettre la validité comparative de la solution facile, voire sa validité tout court. 
Tableau 1 axemple de fabrication des coefficients simples d'Abelson et Tukey (2013) pour $k=7$

\begin{tabular}{lcccccccl}
\hline Étape 1 & 1 & 2 & 3 & 4 & 5 & 6 & 7 & Série de moyenne $(7+1) / 2=4$ \\
Étape 2 & -3 & -2 & -1 & 0 & 1 & 2 & 3 & Soustraction de la moyenne \\
Étape 3 & -12 & -4 & -1 & 0 & 1 & 4 & 12 & Solution 'simple' d'Abelson et Tukey \\
\hline
\end{tabular}

suite jusqu'au groupe 5 avec $200 \mathrm{ml}$. Les résultats moyens des groupes sont :

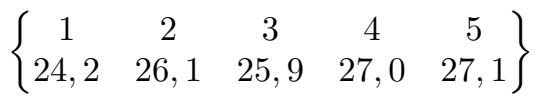

et la valeur estimée de la variance intragroupe $(C M E)$ est 5,50 , avec $N-k=50-5$ degrés de liberté.

La variance observée entre les 5 moyennes, $s^{2}\left(\bar{X}_{j}\right)=$ 1,363 , sert à obtenir le carré moyen des groupes, $C M G=$ $\tilde{n} \cdot s^{2}\left(\bar{X}_{j}\right)=10 \times 1,363=13,63$, cet estimé ayant $k-1=4$ degrés de liberté. On pourrait tester l'hypothèse globale d'une variation systématique non spécifiée, par le quotient $F=C M G / C M E=13,63 / 5,50 \approx 2,478$. Les centiles 95 et 99 de la distribution $F_{4,45}$ étant 2,579 et 3,768, on peut affirmer que le test n'est pas significatif au seuil de $5 \%$.

\section{Procédure selon Barlow et coll. (1972)}

L'hypothèse du chercheur est à l'effet que le café contribue à l'attention et peut augmenter le score de performance des participants. On s'attend donc à ce que les moyennes croissent du groupe 1 au groupe 5. Or, les moyennes obtenues ne répondent pas exactement au modèle de variation monotone, la $2^{e}$ moyenne $(=26,1)$ violant la progression. Il faut donc 'amalgamer' cette valeur ou d'autres valeurs entre elles afin d'obtenir la séquence monotone désirée, ce en veillant à obtenir une solution conforme à variance maximale.

\section{L'amalgamation et le calcul du $C M G^{*}$}

Le procédé d'amalgamation implique au moins deux moyennes, p. ex. la moyenne violatrice et une voisine, et au plus $k-1$ moyennes : l'amalgamation des $k$ moyennes les rendrait toutes égales et donnerait une série de variance nulle. Une série donnée peut aussi contenir plus d'une sous-série violatrice.

L'examen de la série fait ressortir que les valeurs $2^{e}$ et $3^{e}$, soit 26,1 et 25,9, en violent la monotonicité. Plusieurs options sont disponibles, cependant la simple amalgamation des deux valeurs susdites, en les remplaçant par leur moyenne 26,0, s'avère optimale, fournissant la série $\{24,2,26,0,26,0,27,0,27,1\}_{4}^{*}$ conforme au modèle de variation monotone et contenant $r=4$ valeurs distinctes. La variance brute de la série amalgamée est maximale, soit $s^{2}\left(\bar{X}_{j}^{*}\right)=1,358$ (versus la variance initiale $s^{2}\left(\bar{X}_{j}\right)=$ 1,363). Utilisant la formule $3 \mathrm{~b}$, nous calculons :

$$
\bar{E}^{2}=\frac{(5-1) \cdot 10 \cdot 1,358}{(5-1) \cdot 10 \cdot 1.363+(50-5) \cdot 5,50}=0,1799
$$

Cette valeur signifie qu'il y a environ $18 \%$ de variation dans les données associée à la relation monotone d'ordre simple. La consultation des tables présentées en Appendice révèle que pour $d l_{e}=45$ (les degrés de liberté du CME) et $k=5$ (le nombre de moyennes), la valeur significative à $1 \%$ se situe entre 0,1665 et 0,1602 , soit environ $0,1633 .{ }^{4}$ On peut donc conclure que l'hypothèse de variation monotone est confirmée au seuil de $1 \%$; en l'occurrence, la quantité de café bue a un effet cumulatif sur la performance visuomotrice.

\section{Procédures selon Abelson et Tukey (2013)}

Les coefficients $c_{j}$ (6) applicables aux $k=5$ moyennes $\{24,2,26,1,25,9,27,0$ et 27,1$\}$ sont $\{0,8944,0,2010,0,0,2010$ et 0,8944 \}. De là, selon( $5 a)$, le contraste calculé est 2,7747 et, utilisant la valeur $\sum c_{j}^{2} \approx 1,6807$ et $n=10$, nous obtenons par (5b) le $C M C=10 \times 2,77472 / 1,6807 \approx 45,807 .{ }^{5} \mathrm{Le}$ test $F$ résultant, soit $F=C M C / C M E=45,807 / 5,50 \approx$ 8,328 est de probabilité 0,006 selon $F_{1 ; 45}$, confirmant significativement l'allure monotone des moyennes au seuil de 0,01 .

Par la solution '4-2' proposée d'Abelson et Tukey (ibid.) basée sur les coefficients modifiés du polynôme linéaire de premier degré, soit $-8,-2,0,2$ et 8 , le lecteur pourra vérifier, qu'on obtient $C M C=10 \times 25,02 / 136 \approx 45,956$ et $F=45,956 / 5,50 \approx 8,356$, à significativité comparable à celle de la solution 'géométrique' traitée auparavant.

\section{Un second exemple, portant sur des proportions}

Pour notre second exemple, et afin d'y introduire le lecteur, l'analyse traitera de proportions, où l'on se demandera si, d'une condition à l'autre ou d'un groupe à l'autre, les proportions ont évolué de façon régulière.

L'exemple, fictif, concerne la proportion de femmes qui occupent le siège de président-directeur-général dans des entreprises du Québec roulant sur un budget annuel situé dans l'intervalle de 10 à 50 millions de dollars. Les données couvrent les années de 2000 à 2019, et sont regroupées

4. Voir l'Appendice pour la solution à différents contextes d'interpolation.

5. Les coefficients et sommes de carrés du tableau A5 sont présentés selon une précision de 3 décimales. Le recours commode à ces constantes approximatives n'a pas d'impact invalidant sur le résultat. 
Tableau 2 घ Présence de femmes dans les postes de haute gestion (données brutes fictives)

\begin{tabular}{cllllllllll}
\hline Années & 2000 & 2002 & 2004 & 2006 & 2008 & 2010 & 2012 & 2014 & 2016 & 2018 \\
& 2001 & 2003 & 2005 & 2007 & 2009 & 2011 & 2013 & 2015 & 2017 & 2019 \\
\hline Femmes $x$ & 7 & 6 & 7 & 10 & 9 & 10 & 11 & 13 & 17 & 16 \\
Tous $n$ & 75 & 79 & 76 & 80 & 80 & 84 & 82 & 92 & 86 & 83 \\
\hline
\end{tabular}

par paires d’années. Le tableau 2 présente ces données. On constate qu'il y aurait une certaine croissance irrégulière du nombre de semi-grandes entreprises au Québec. Qu'en est-il de la proportion de femmes qui sont à leur tête ${ }^{6}$

Le tableau 3 prolonge le tableau 2, les données étant cette fois codifiées en y ajoutant quelques lignes qui serviront à l'analyse.

La variable 'proportion' $p$ tout comme son numérateur $x$ font bande à part dans les traitements statistiques, en raison de leurs propriétés numériques particulières. La proportion par exemple a des valeurs bornées entre 0 et 1 et, pour une proportion de référence $\pi$, les proportions associées, de moyenne individuelle $p_{i}$, fluctuent selon des variances qui dépendent de leurs moyennes, c.à-d. des $x_{i}$. De plus, leurs indices de forme, l'asymétrie $\left(\gamma_{1}\right)$ et l'aplatissement $\left(\gamma_{2}\right)$, en sont aussi affectés. Ces deux caractéristiques éloignent la variable $p$ des conditions de normalité généralement exigées pour l'analyse. Peut-on remédier à cet inconvénient de manière à traiter correctement la série de proportions ci-dessus?

Laurencelle (2021a, 2021b; voir aussi Laurencelle et Cousineau, à paraître) propose pour ces analyses deux transformations mathématiques d'une proportion, dont l'origine remonterait à R. A. Fisher, celle d'Anscombe (1948), notée ici $y_{A n s}$, et celle de Chanter (1975), notée $y_{C h a}$. Ces transformations sont :

$$
\begin{gathered}
y_{A n s}(x, n)=\sin ^{-1} \sqrt{\frac{X+3 / 8}{n+3 / 4}} ; \\
\operatorname{var}(x) \approx 1 /(4 n+2), \\
y_{C h a}(x, n)=x=\sin ^{-1} \sqrt{\frac{1}{4 n}+\left(1-\frac{1}{2 n}\right) \frac{X}{n}} ; \\
\operatorname{var}(x) \approx 1 / 4 n,
\end{gathered}
$$

la fonction trigonométrique " $\sin ^{-1}$ " ou " arcsin " étant donnée en radians. La seconde formule, $y_{C h a}$, est plutôt recommandée pour des ensembles de proportions comportant de petites tailles $n$ inégales (voir Laurencelle, 2021b). Ici, les tailles, au dénominateur des proportions, étant inégales mais toutes suffisamment fortes, c.-à-d. > 30, on peut utiliser la fonction d'Anscombe.

Illustrons le calcul pour la période 1, ou les années 2000-2001. Les femmes recensées sont au nombre de $x=7$, pour un nombre total de dirigeants (incluant les hommes) de $n=75$, d'où la proportion $p=7 / 75 \approx 0,093$, apparaissant au tableau 3. La valeur transformée selon Anscombe s'obtient par :

$$
y_{A n s}(7,75) \approx \sin ^{-1} \sqrt{\left(7+\frac{3}{8}\right) /\left(75+\frac{3}{4}\right)} \approx 0,3173
$$

les valeurs transformées occupant l'intervalle dans 0 à $\approx$ $1,571(=\pi / 2)$ pour $p$ dans 0 à 1 . Le lecteur aura noté aussi que la variance d'erreur (ou incertitude) attachée à chaque valeur transformée (voir les formules associées à (7) et (8)) ne dépend pas de la valeur de la proportion mais seulement de sa taille $n$, ce qui en principe la rend insensible à la valeur naturellement fluctuante du numérateur $x$, au contraire de la proportion brute $p$.

L'analyse de variance simple de $k$ proportions indépendantes. Hors de tout modèle de variation, y at-il des différences globales entre les 10 proportions retenues. Ici, le calcul est relativement simple. Chaque proportion considérée constitue une valeur unitaire et représente donc une seule donnée pour l'analyse, sans considération de sa taille $n$, laquelle intervient seulement pour le calcul du terme d'erreur. Ainsi, par une adaptation de la formule (2), nous avons $C M G=1 \cdot s^{2}\left(y_{A n s_{j}}\right)=0,00338$. Quant au terme d'erreur ou $C M E$, la variance d'erreur considérée, c.-à-d. la variance aléatoire des valeurs transformées de chaque proportion, est réputée constante en vertu de la transformation appliquée, ne dépendant que de la taille $n$, au dénominateur de chaque proportion. Pour chacune, utilisant la fonction de transformation d'Anscombe, la variance d'erreur selon $n_{j}$ serait $\sigma_{e}^{2}=1 /\left(4 n_{j}+2\right)$. L'estimation du terme d'erreur global, le $C M E$, se fait alors en combinant les différentes proportions, soit selon la moyenne arithmétique des estimés d'erreur de chacun, soit par la formule $1 /(4 \tilde{n}+2), \tilde{n}$ étant encore la moyenne harmonique des $k$ tailles $n_{j}$. Dans le cas des données du tableau 3, les deux approches donnent la même valeur sur

6. La variation du nombre absolu de femmes 'au pouvoir' peut aussi être soumise au test. Le khi2 de 11,925 évalué contre une hypothèse générale de variation systématique n'atteint pas sa valeur critique $\chi_{10-1}^{2}(0,05)=16,92$, mais celui obtenu après amalgamation contre le modèle de variation monotone y parvient, avec une valeur de 11,783 contre $\bar{\chi}_{10}^{2}(0,005)=11,768$. La puissance accrue grâce au recours à une contre-hypothèse spécifique plutôt que générale n'est plus à démontrer. D’autre part, l’application de la variable $\bar{\chi}^{2}$ de Barlow et coll. au présent contexte reste à valider. 
Tableau 3 Présence de femmes dans les postes de haute gestion (données traitées)

\begin{tabular}{cllllllllll}
\hline Période & 1 & 2 & 3 & 4 & 5 & 6 & 7 & 8 & 9 & 10 \\
\hline$p=x / n$ & 0,093 & 0,076 & 0,092 & 0,125 & 0,113 & 0,119 & 0,134 & 0,141 & 0,198 & 0,193 \\
$y_{\text {Ans }}$ & 0,3173 & 0,2866 & 0,3152 & 0,3666 & 0,34772 & 0,35740 &, 37989 & 0,3895 & 0,4640 & 0,4580 \\
$y *$ & 0,30915 & 0,30915 & 0,3152 & 0,35715 & 0,35715 & 0,3574 & 0,3798 & 0,3895 & 0,4610 & 0,4610 \\
\hline
\end{tabular}

cinq décimales, soit $C M E \approx 0,00305$.

Comme en analyse de variance classique, le $C M G$ a $k-1$ degrés de liberté parce qu'il met en comparaison $k$ moyennes. Quant au terme d'erreur, mathématiquement déterminé par la transformation, il est fixe et n’a pas de distribution (correspondant ainsi à une statistique estimée sur un nombre infiniment grand de degrés de liberté. Deux tests sont alors possibles : un test $F=C M G / C M E$ jugé d'après la loi $F(k-1, \infty)$, ou son équivalent $k h i 2=$ $(k-1) \cdot C M G / C M E$ jugé d'après la loi $\chi^{2}(k-1)$. Prenant indifféremment ce second moyen nous obtenons khi $2=(10-1) \cdot 0,00338 / 0,00305 \approx 9,973$, de probabilité extrême $\approx 0,353$, les variations observées ne ressortant pas remarquables.

Le test de variation global de nos 10 proportions s'avère donc négatif. Mais s'y trouve-t-il tout de même une tendance réelle à une progression croissante des proportions?

\section{Procédure selon Barlow et coll. (1972)}

$\mathrm{Au}$ tableau 3, dans la rangée titrée $y_{A n s}$, la croissance monotone attendue des valeurs se voit contrariée en quelques endroits. Une recherche patiente ou bien l'exploitation d'un programme informatique (voir en appendice le texte d'un programme composé à ce propos en langage Pascal-Delphi) fournira la série amalgamée optimale, qui paraît au même tableau, titrée $y *$ en dernière ligne. Trois groupes de proportions (inscrites en caractères italiques) ayant été trouvées, la série résultante, qui contient $r=7$ valeurs distinctes, a comme variance brute $s^{2}\left(y_{A n s}^{*}\right)=$ 0,00330 .

Dans le cas de proportions, ici des proportions transformées, chaque valeur est considérée unitaire. c.-à-d. de taille 1. Ainsi, en recourant aux formules (3a) ou (3b), la taille $\tilde{n}$ est supposée de valeur 1. Par exemple, prenant l'expression (3a), le carré moyen des conditions, $C M G^{*}$ s'obtient par $s^{2}\left(\bar{X}_{j}^{*}\right) \cdot(k-1) /(r-1)$, ici $0,00330 \times(10-$ $1) /(7-1) \approx 0,00494$. Quant au terme d'erreur, c'est le même qu'on appliquerait ici à l'analyse de variance des proportions : ci-dessus, nous obtenions $C M E=0,00305$. La variance d'erreur étant ici théoriquement fixée, c'est la version (4) du test de variation monotone qui s'applique, donnant ici $\bar{\chi}^{2}=(7-1) \cdot 0,00494 / 0.00305 \approx 9,720$. Les tables de l'Appendice donnent 8,145 et 10,216 pour valeurs critiques aux seuils de 0,025 et 0,01 respectivement : l'interpolation sur $\ln p$ indique une probabilité de $\approx 0,012$.
Nos données démontrent donc une croissance significative de la proportion de femmes à la direction des semi-grandes entreprises au Québec.

\section{Procédures selon Abelson et Tukey (2013)}

Aux $k=10$ proportions de notre second exemple, les coefficients $c_{j}$ (6) d'Abelson et Tukey sont $0,9487,-0,3162,-0,1842,-0,1001,-0,0319$ + le miroir positif des précédents. Le contraste brut (5a), ici, soit $-0,9487 \times 0,3173-0,1842 \times 0,2866+\cdots+0,9487 \times$ 0,4580 donne 0,20490 et, se rappelant qu'ici, pour les proportions, nous avons $\tilde{n}=1$, le $C M C$ (5b) vaut $1 \times 0,204902 / 2,08996 \approx 0,02009$. Le test à appliquer, pour le quotient $C M C / C M E=0,02009 / 0,00305 \approx$ 6,584 , peut s'inscrire ici à la fois dans un $F_{1, \infty}$ ou un $\chi_{1}^{2}$, sa probabilité extrême étant $\approx 0,010$.

L'approximation " 4-2 » des auteurs produit ici un quotient de 6,816 de probabilité 0,009. Par ailleurs, le recours à un contraste reflétant une simple droite (ou polynôme de degré 1) résulterait pour sa part en un quotient $C M L / C M E \approx 8,521$, de probabilité 0,004. Ce dernier test, à significativité plus forte, prouve que la série étudiée comporte une tendance ferme à l'accroissement : prouvet-il que cette tendance est monotone? Nous discutons de cette question plus bas.

\section{Le traitement de modèles d'analyse sur mesures répétées}

La comparaison de l'effet de conditions expérimentales de plus en plus exigeantes, de doses de traitement médicinal, de niveaux croissants d'exercice sur le même groupe de participants et tutti quanti se prête aussi à l'analyse de variation monotone, à l'instar de l'analyse en polynômes orthogonaux basée sur un modèle de variation linéaire stricte. La documentation citée, notamment Laurencelle (2021b) et Laurencelle et Cousineau (soumis), informe sur la question (voir aussi Mukerjee, 1988). Exprimé sténographiquement, la solution consiste simplement à réduire le $C M E$ en le multipliant par $\left(1-\alpha_{1}\right)$, où $\alpha_{1}=$ $\alpha /[k-(k-1) \cdot \alpha]$, et $\alpha$ est le coefficient de consistance interne de Cronbach (1951). Pour l'analyse de proportions, l'estimation du coefficient $\alpha$ se fait à partir du tableau des données brutes ( 0 ou 1 ) des participants. 


\section{En conclusion}

Les techniques d'analyse présentées dans cet essai sont aujourd'hui encore peu connues et il est rare d'en constater l'application dans les publications scientifiques. Pourtant, selon notre expérience, nombre de problématiques de recherche laissent affleurer l'hypothèse de variation monotone : comparaison de l'effet de doses croissantes d'un médicament ou d'un traitement, évolution du phénomène observé selon différents moments successifs, efficacité comparée de niveaux d'une variable indépendante à valeurs croissantes. Tous ces protocoles d'étude sont des candidats à fonder une hypothèse de variation monotone. Ils sont occasionnellement traités par un test de croissance linéaire, nous l'avons évoqué dans notre exposé. Cependant la réponse donnée par ce test reste équivoque : une composante linéaire (de premier degré) significative indique que la série considérée présente une variation linéaire sérieuse, mais ce test n'exclut pas qu'une autre composante, non-linéaire celle-là, soit présente et que finalement la variation étudiée ne soit pas monotone. La plupart du temps, l'analyse se fera (encore aujourd'hui) par la comparaison deux à deux des paliers successifs de la série, voire du test d'un $j^{e}$ palier comparé au palier 1, espérant trouver un premier palier significatif à un seuil de significativité donné, puis d'autres paliers plus distants à des seuils de plus en plus exigeants. Cette procédure, très coûteuse en probabilité et sujette à une police de contrôle de type Bonferroni, est une manœuvre mathématiquement illogique, non concluante et surtout de puissance affaiblie. Par opposition, la démonstration d'une variation monotone significative par un seul test global est très puissante (Barlow et coll., 1972) et constitue une preuve de la tendance générale des données qui s’applique tout le long de la série analysée.

Le test substitutif avancé par Abelson et Tukey (2013), évidemment très puissant lui aussi, tient peut-être sa surcharge de puissance par l'artifice apparent de son moyen, celui de se centrer surtout sur les deux extrémités de la série, exagérant leur poids en variance au moyen des coefficients appliqués et pouvant y créer un semblant de variation monotone. ${ }^{7}$

Si la série à analyser se présente déjà en variation régulière, sans violation de monotonicité, l'une ou l'autre méthode convient, voire l'analyse de variance toute simple. Il resterait à déterminer la puissance comparée de ces trois approches.

\section{Références}

Abelson, R. P. \& Tukey, J. W. (2013). Efficient utilization of non-numerical information in quantitative analyses general theory and the case of simple order. Annals of Mathematical Statistics, 34, 1347-1369.

Anscombe, F. J. (1948). The transformation of Poisson, binomial and negative-binomial data. Biometrika, 35, 246-254.

Barlow, R. E., Bartholomew, D. J., Bremner, J. M. \& BRUNK, H. D. (1972). Statistical inference under order restrictions. Wiley : New York.

BARTHOLOMEW, D. J. (1959a). A test of homogeneity for ordered alternatives. Biometrika, 46, 36-48.

BARTholomew, D. J. (1959b). A test of homogeneity for ordered alternatives II. Biometrika, 46, 328-335.

BARTHOLOMEW, D. J. (1961). Ordered tests in the analysis of variance. Biometrika, 48, 325-332.

CHANTER, D. O. (1975). Modifications of the angular transformation. Journal of the Royal Statistical Society Series B (Applied Statistics), 24, 354-359.

CRONBACH, L. J. (1951). Coefficient alpha and the internal structure of tests. Psychometrika, 16, 297-334.

DRAPER, N. \& SMITH, H. (1981). Applied regression analysis (2e édition). New York : Wiley.

HAYTER, A. J. (1990). A one-sided studentized range test for testing against a simple ordered alternative. Journal of the American Statistical Association, 85(778-785), 778-785.

Jonckeere, A. R. (1954). A distribution-free k-sample test against ordered alternatives. Biometrika, 41, 133-145.

LAURENCELlE, L. (1993). Deux tests de variation monotone pour l'analyse de variance. Lettres statistiques, 9, 6991.

LAURENCELLE, L. (2002). Tables statistiques expliquées et appliquées (2e édition). Québec : Le Griffon d'argile.

LAURENCELlE, L. (2014). Les tests de variation monotone et la détermination de la dose effective. Le colloque Méthodes Quantitatives en Sciences Humaines, Université d'Ottawa, Ottawa, le 6 juin 2014.

LAURENCELLE, L. (2017). The unweighted 'harmonic mean' solution for unbalanced anova designs : A detailed argument [L'analyse de variance pour des groupes inégaux et la solution du $\mathrm{n}$ harmonique : une question d'équité]. The Quantitative Methods for Psychology, 13, 95-104.

LAURENCELLE, L. (2021a). Étude comparative de trois fonctions de transformation normalisante d'une proportion (Anscombe, 1948 - Freeman-Tukey, 1950 - Chanter, 1975. The Quantitative Methods for Psychology, 17, 166-197.

LAURENCELle, L. (2021b). Le traitement statistique des proportions incluant l'analyse de variance, avec des exemples /The statistical handling of proportions

7. Tout se passe comme si, en endossant d'emblée un modèle de variation linéaire de la série, on en exagérait l'impact des extrémités afin d'en accroître encore les chances de significativité, ce en ignorant relativement ses variations plus centrales. 
including analysis of variance, with worked out examples. The Quantitative Methods for Psychology, 17, 272-285.

LAURENCELLE, L. (2021c). Les distributions multinomiales, leur mesure par les tests khi2 et G, leur approximation par la loi chi-2 et l'analyse des tableaux de fréquences par le test G. The Quantitative Methods for Psychology, 17, 375-394. doi :10.20982/tqmp.17.4.p375

LAURENCELle, L. \& CousineaU, D. (soumis). Analysis of proportions using arcsine transform with any experimental design.
MuKeRJEE, H. (1988). Order restricted inference in a repeated measures model. Biometrika, 75, 616-617.

Ruben, H. (1954). On the moments of order statistics in samples from normal populations. Biometrika, 41, 220-227.

WineR, B. J., Brown, D. R. \& MicheLs, K. M. (1991). Statistical principles in experimental design (3e édition). New York : McGraw Hill. 


\section{Appendice}

\section{Formules et calcul des valeurs critiques des tests $\bar{\chi}^{2}$ et $\bar{E}^{2}$}

Le chapitre 3 du volume Statistical Inference under Order Restrictions de Barlow et coll. (1972) récapitule la théorie de distribution des tests de variation monotone. Nous présentons ici les formules fondamentales de cette théorie, en développant le cas spécifique auquel réfère l'hypothèse dite d'ordre simple. Rappelons que nous voulons tester l'hypothèse nulle de non-différence entre $k$ moyennes, versus la contre-hypothèse d'une variation monotone de celles-ci. Le paramètre $k$ dénote encore de nombre total de moyennes de la série et $r$, le nombre de moyennes encore distinctes après 'amalgamation'.

Les théorèmes (3.1) et (3.2) dans Barlow et coll. (p. 126-127) proposent les équations déterminant les valeurs critiques, respectivement pour les statistiques $\bar{\chi}^{2}$ et $\bar{E}^{2}$. Si l'on possède la variance paramétrique $\sigma_{e}^{2}$, alors la distribution de $\bar{\chi}^{2}$ s'applique; elle sert aussi à interpoler une valeur critique utilisant un terme d'erreur estimé sur un (ou plusieurs) échantillon(s) dont les degrés de liberté $\left(d l_{e}\right)$ déborderaient le nombre maximal (250) tabulé (voir plus bas). Sa distribution dé-cumulative est :

$$
\operatorname{Pr}\left\{\bar{\chi}^{2} \geq C\right\}=\sum_{r=2}^{k} P_{h}(k, r) \cdot \operatorname{Pr}\left\{\chi_{r-1}^{2} \geq C\right\}, C>0 ;
$$

et :

$$
\operatorname{Pr}\left\{\bar{\chi}^{2}=0\right\}=P_{h}(k, 1) .
$$

Cette probabilité extrême, dont sont tirées des valeurs critiques correspondant à des seuils de probabilité donnés, est ainsi une moyenne pondérée de variables $\chi^{2}$, les poids $P_{h}$ variant selon l'hypothèse de recherche envisagée .

Si on ne dispose pas de la variance paramétrique $\sigma^{2}$, on peut ordinairement en obtenir une estimation, $C M E$, basée $\operatorname{sur} Q$ degrés de liberté. Dans ce cas, l'analyse de variance s'applique, et nous obtenons le quotient $\bar{E}^{2}$, ayant pour distribution dé-cumulative :

$$
\operatorname{Pr}\left\{\bar{E}^{2} \geq C\right\}=\sum_{r=2}^{k} P_{h}(k, r) \cdot \operatorname{Pr}\left\{B_{1 / 2(r-1), 1 / 2(d l e+k-r)} \geq C\right\}, C>0 ;
$$

et :

$$
\operatorname{Pr}\left\{\bar{E}^{2}=0\right\}=P_{h}(k, 1),
$$

la notation $B_{a, b}$ désignant la loi Bêta (à la base de l'analyse de variance) avec deux paramètres. Comme on voit, la probabilité cherchée ici comme plus haut est une somme pondérée de probabilités, les pondérations notées $P_{h}$, référant à l'hypothèse de variation élue. Pour l'hypothèse d'une variation monotone des moyennes, les auteurs (ibid., p. 145) donnent :

$$
\begin{aligned}
P h(1, k) & =1 / k, \\
P h(k, k) & =1 / k ! \\
\text { et } P h(k, r) & =\frac{1}{k} P(r-1 . k-1)+\frac{k-1}{k} P_{h}(r, k-1) .
\end{aligned}
$$

\section{Tables de valeurs critiques des statistiques $\bar{E}^{2}$ et $\bar{\chi}^{2}$ et leur lecture}

Les tableaux A1 à A4 offerts dans les pages qui suivent présentent les valeurs critiques de la statistique $\bar{E}^{2}$ selon les seuils de significativité respectifs $\alpha$ de 0,005, 0,01, 0,025 et 0,05, ce pour des degrés de liberté (dle) du $C M E$ (le terme d'erreur, au dénominateur des formules) de 2 à 250 selon 2(1)20(2)50(5)100(25)200 et 250, incluant l'infini (noté $\infty$, en bas de tableau. Par exemple, pour une série de $k=8$ valeurs, qualifiée d'un terme d'erreur ayant 17 dle au seuil $\alpha$ de 0,01, la valeur critique de $\bar{E}^{2}$ à appliquer, au tableau A2, est 0,3614 . Au bas de chaque tableau, le lecteur trouvera les valeurs critiques correspondantes convenant aux cas où le terme d'erreur paramétrique $\left(\sigma_{e}^{2}\right)$ est connu, tel qu'approprié pour le traitement d'une série de proportions exposé ci-dessus.

Dans le cas d'un terme d'erreur dont la valeur des dle est intercalée entre les valeurs tabulées, par exemple dle $=225$ (entre 200 et 250), l'interpolation est possible. L'interpolation linéaire simple (sur dle) est moins sûre ici, sauf dans le cas d'un intervalle de valeurs dle très petit, soit 2 ou 3. Pour un grand intervalle et surtout lorsque la valeur cherchée 
s'y trouve au milieu, comme dans notre exemple, la méthode plus simple recommandée est l'interpolation harmonique, c.-à-d. sur 1/dle plutôt que dle. Un raffinement suggéré, justifié par l'allure exponentielle de la courbe de distribution de $\bar{E}^{2}$, consiste à interpoler le logarithme de la valeur critique sur $1 /$ dle.

Cas 1 : Interpolation d'une valeur critique. Prenons pour exemple le cas de $k=8$ valeurs soumises à un dle de 225, à tester au seuil $\alpha$ de 0,01. La valeur cherchée a un dle logé au centre de l'intervalle 200-250, entre les valeurs critiques respectives 0,0460 et 0,0372 au seuil prescrit de 0,01 (voir tableau A2). Nous obtenons 0,0416 pour l'interpolation linéaire simple $[0,0460+(0,0372-0,0460) \times(225-200) /(250-200)], 0,0411$ pour l'interpolation harmonique $[0,0460+(0,0372-0,0460) \times(1 / 225-1 / 200) /(1 / 250-1 / 200)]$ et 0,0409 pour la version harmonico-logarithmique de l'interpolation, $\exp ((\ln 0,0460+(\ln 0,0372-\ln 0,0460) \times(1 / 225-$ $1 / 200) /(1 / 250-1 / 200))$, la plus sûre à notre avis.

Cas 2 : Estimation d'une probabilité exacte. Par ailleurs, pour un dle donné et une valeur critique observée de $\bar{E}^{2}$, l'utilisateur intéressé peut estimer la probabilité extrême exacte de son résultat, en l'interpolant entre le seuil $\alpha$ franchi et le seuil $\alpha$ plus exigeant, la relation entre le logarithme de la probabilité et la valeur $\bar{E}^{2}$ correspondante étant quasi linéaire. Encore pour notre exemple, avec $k=8$ et $d l e=200$, supposons que la valeur $\bar{E}^{2}$ obtenue est de 0,0390. Les valeurs critiques affichées aux tableaux A2 et A3 sont 0,0460 (pour $p=0,01$ ) et 0,0366 (pour $p=0,025$ ) : le résultat est donc significatif au seuil $\alpha=0,025$ mais pas au seuil $\alpha=0,01$. L'interpolation sur logarithme de $p$ est $\exp [\ln 0,01+(\ln 0,025-\ln 0,01) \times(0,0390-$ $0,0366) /(0,0460-0,0366)] \approx 0,0198$, une estimation précise à $\pm 0,0002$.

Cas 3 : Extrapolation au-delà d'un dle $=250$. Supposons maintenant une situation $k=8, \alpha=0,1$ et $d l e=400$, donc dans la zone non tabulée sise entre $d l e=250$ et $\infty$. Une estimation valable mais grossière consisterait simplement à diviser la valeur critique $\bar{\chi}^{2}$ présentée par dle. Ici, la valeur au tableau A2 est 9,638, d'où la valeur critique estimée serait $\bar{E}^{2}=9,638 / 400 \approx 0,0241$. Pour une estimation plus juste, l'interpolation harmonique entre $d l e=250$ et $\infty$ reste encore préférable, se rappelant que $1 / \infty=0$. Par exemple, pour un $d l e=400$, toujours avec $k=8$ valeurs et au seuil de 0,01 , le tableau A2 présente $\bar{E}^{2}=0,0372$ à $d l e=250$ et $\bar{\chi}^{2}=9,638$ pour dle $=\infty$. Le calcul se base sur les quantités $t_{250}=250 \times 0,0372=9,3000$ et $t_{\infty}=9,638$ pour déterminer d'abord $t_{d l e}=d l e \times \bar{E}_{d l e}^{2}$ par $t_{d l e}=t_{250}-\left(t_{\infty}-t_{250}\right)\left(\frac{\text { dle-250 }}{\text { dle }}\right)$, puis $\bar{E}_{\text {dle }}^{2}=t_{\text {dle }} /$ dle $=9,1733 / 400 \approx 0,0229$. 
Tableau A1 - Valeurs critiques des tests $\bar{E}^{2}$ et $\bar{\chi}^{2}$ au seuil $\boldsymbol{\alpha}=\mathbf{0 , 0 0 5}$

\begin{tabular}{|c|c|c|c|c|c|c|c|c|c|c|c|c|c|c|}
\hline dle / $\mathrm{k}$ & 2 & 3 & 4 & 5 & 6 & 7 & 8 & 9 & 10 & 11 & 12 & 13 & 14 & 15 \\
\hline 2 & 9801 & ,9751 & ,9584 & ,9327 & ,9013 & 8673 & ,8330 & ,7994 & ,7673 & ,7369 & ,7084 & ,6817 & ,6567 & ,6334 \\
\hline 3 & 9192 & 9200 & 9019 & ,8758 & 8460 & ,8149 & ,7840 & ,7540 & ,7254 & 6983, & 6728 & ,6488 & 6263 & ,6052 \\
\hline 4 & 8413, & 8542 & 8410 & ,8188 & ,7929 & ,7658 & ,7387 & ,7123 & 6870, & 6629, & 6401, & ,6186 & 5983, & ,5793 \\
\hline 5 & 7648, & ,7892 & ,7824 & ,7653 & 7438, & ,7206 & 6972, & 6741 & 6518, & 6305, & 6101, & ,5909 & ,5726 & ,5553 \\
\hline 6 & 6961 & ,7293 & ,7286 & ,7162 & 6989, & 6795 & 6594 & 6394 & 6197, & 6008, & ,5826 & ,5653 & ,5488 & ,5331 \\
\hline 7 & 6363 & 6757, & 6800 & 6719 & 6583, & 6422 & 6250 & 6076 & ,5904 & ,5736 & 5573, & ,5418 & ,5268 & ,5126 \\
\hline 8 & ,5846 & 6281 & ,6364 & 6319 & 6215, & 6083 & 5937, & ,5786 & ,5635, & ,5486 & ,5340 & ,5200 & ,5065 & 4935 \\
\hline 9 & 5399 & 5861 & ,5975, & ,5960 & 5883, & ,5775, & 5652 & ,5521 & 5388, & 5255, & ,5125, & 4998 & 4875, & 4757 \\
\hline 10 & 5011, & ,5488 & ,5627 & ,5635 & 5581, & ,5494 & 5390 & ,5277 & ,5160 & ,5042 & 4925, & 4811 & 4699, & 4591 \\
\hline 11 & 4672 & 5157, & 5313, & ,5342 & 5307, & ,5238 & 5151, & ,5053, & 4950, & 4845, & 4740, & 4637, & 4535, & ,4436 \\
\hline 12 & 4374, & 4861 & ,5031 & ,5076 & 5057, & ,5004 & 4931, & ,4846 & 4756, & 4663, & 4568, & ,4474 & 4382, & 4291 \\
\hline 13 & 4111, & 4596 & ,4776 & ,4834 & 4828, & ,4788 & 4728 & 4655, & 4576, & 4493, & 4408, & ,4323 & 4238, & 4155 \\
\hline 14 & 3876 & 4357, & 4545 & ,4613 & 4619, & ,4590 & 4541, & ,4478 & 4409, & 4334, & 4258 & ,4180 & 4103 & 4027 \\
\hline 15 & 3666 & 4141 & 4334 & ,4410 & 4426 & ,4407 & 4367, & 4314 & 4253, & 4187, & 4118 & 4047 & 3977, & ,3906 \\
\hline 16 & 3478, & 3945, & 4141 & 4224 & 4248, & 4238, & 4206 & 4161 & 4107, & 4048, & 3986, & ,3922 & 3857, & ,3793 \\
\hline 17 & 3307 & ,3766 & 3965 & ,4053 & 4084, & 4080 & 4056, & 4018 & 3971 & 3919, & 3862 & ,3804 & 3745, & ,3686 \\
\hline 18 & ,3152 & ,3603 & ,3802 & ,3895 & 3931 & ,3934 & 3916 & ,3884 & 3844 & 3797 & ,3746 & ,3693 & 3639, & ,3584 \\
\hline 19 & 3011, & 3452 & ,3652 & ,3748 & ,3790 & 3798 & 3785, & ,3759 & ,3724 & ,3682 & 3637, & ,3588 & 3539, & ,3488 \\
\hline 20 & 2882 & 3314 & 3513 & ,3612 & 3658 & 3671 & 3663 & ,3642 & 3611, & 3574 & 3533, & ,3489 & 3443, & 3397 \\
\hline 22 & 2653, & 3067, & ,3264 & ,3367 & 3419, & 3439 & 3440 & 3427 & 3405, & 3376 & 3343, & ,3306 & ,3268 & ,3228 \\
\hline 24 & 2458, & 2855, & ,3048 & ,3153 & ,3209 & 3235, & ,3242 & ,3236 & 3221 & 3198, & 3172 & ,3141 & 3109, & ,3075 \\
\hline 26 & 2290 & 2669 & 2858 & 2963 & 3023, & 3054 & 3066 & 3065 & 3055, & 3038, & 3017, & 2992 & 2964 & 2935 \\
\hline 28 & 2143, & 2506 & 2690 & ,2796 & 2857, & 2891 & 2907, & 2911 & 2905, & 2893, & 2876, & 2856, & 2833, & 2808 \\
\hline 30 & 2013 & 2362 & 2541 & ,2646 & 2708, & 2745 & 2764 & 2771 & 2770 & 2761 & 2748, & 2732, & 2712, & 2691 \\
\hline 32 & 1899, & 2233, & 2407 & 2511 & 2574 & 2613 & 2635, & 2645 & 2646 & 2641 & 2631 & ,2618 & 2602 & ,2583 \\
\hline 34 & 1796, & 2117, & 2287 & ,2389 & 2453, & 2493 & 2517, & 2529 & 2533, & 2530 & 2524 & ,2513 & 2500 & ,2484 \\
\hline 36 & 1704, & 2013, & 2178 & 2278 & 2342 & 2383 & 2409 & 2423 & 2429 & 2429 & 2424 & ,2416 & 2405, & ,2392 \\
\hline 38 & 1621, & 1919, & 2079 & 2177 & 2241, & 2283 & 2309, & 2325 & 2333, & 2335, & 2332 & ,2326 & 2317, & ,2306 \\
\hline 40 & 1546, & 1833, & 1988 & 2085 & 2148 & 2190 & 2218 & 2235 & 2244 & 2248 & 2247 & ,2243 & 2236 & ,2227 \\
\hline 42 & 1477, & 1754 & 1905, & 2000 & 2063 & 2105 & 2133 & 2152 & 2162 & 2167 & 2168 & ,2165 & 2160 & ,2152 \\
\hline 44 & 1414, & 1682, & 1829 & 1922 & 1984, & 2026 & 2055, & 2074 & 2086, & 2092 & 2094 & ,2093 & 2089, & 2083 \\
\hline 46 & 1357, & 1615, & 1758 & 1849 & 1911, & 1953, & 1982, & 2002 & 2015, & 2022 & 2025, & ,2025 & 2023 & 2018 \\
\hline 48 & 1303, & 1554 & 1693 & ,1782 & 1843, & 1885, & 1914, & 1935, & 1948, & 1957, & 1961, & 1962 & 1960, & 1956 \\
\hline 50 & 1254, & 1497, & 1632 & ,1720 & 1779, & 1821, & 1851, & 1872 & 1886, & 1895, & 1900, & 1902 & 1901, & 1899, \\
\hline 55 & 1146, & 1371, & 1498 & 1581, & 1639, & 1680, & 1710, & 1731, & 1746, & 1757, & 1764, & 1768 & 1769, & 1768 \\
\hline 60 & 1055, & 1265, & 1384 & 1463 & 1518, & 1559, & 1588, & 1610 & 1626, & 1638, & 1646, & 1651 & 1654, & 1654 \\
\hline 65 & 0977, & 1174, & 1287, & 1362 & 1415, & 1454 & 1483, & 1505, & 1521, & 1533, & 1542, & 1549, & 1553, & 1555, \\
\hline 70 & 0910, & 1095, & 1202 & 1273, & 1324, & 1362 & 1391, & 1412, & 1429, & 1442, & 1451, & ,1458 & 1463, & 1466 \\
\hline 75 & 0852, & 1026, & 1127, & 1195, & 1244, & 1281, & 1309, & 1331, & 1347, & 1360, & 1370, & ,1378 & 1383, & 1387 \\
\hline 80 & 0801 & ,0965 & 1062 & 1127 & 1174, & 1209, & 1236, & 1258 & 1275, & 1288, & 1298, & ,1306 & 1312, & 1316, \\
\hline 85 & 0755, & 0911 & 1003 & 1065 & 1111, & 1145, & 1172, & 1193, & 1209, & 1222 & 1233, & ,1241 & 1247, & 1252 \\
\hline 90 & ,0714 & 0863 & ,0951 & 1010 & 1054, & 1087 & 1113, & 1134 & 1150, & 1163, & 1174, & ,1182 & 1189, & 1194 \\
\hline 95 & ,0678 & ,0820 & ,0903 & ,0961 & 1003, & 1035, & 1060, & 1080, & 1097, & 1109, & 1120, & ,1129 & 1135, & 1141 \\
\hline 100 & ,0645, & ,0780 & ,0861 & ,0916 & 0957, & ,0988 & 1012, & 1032, & 1048, & 1061, & 1071, & 1080 & 1087, & 1092 \\
\hline 125 & 0519, & ,0630 & ,0696 &, 0742 & 0777, & ,0804 & ,0825, & ,0843 & 0857, & 0869 & 0879, & ,0888 & 0895, & 0901 \\
\hline 150 & ,0434 & ,0528 & ,0584 & ,0624 & ,0654 & ,0677 & ,0696 & ,0712 & ,0725, & ,0736 & ,0745, &, 0754 & ,0761 & ,0767 \\
\hline 175 & 0373, & ,0454 & ,0503 & 0538 & ,0565, & ,0585 & ,0602 & ,0617 & ,0628 & ,0639 & ,0647 &, 0655 & ,0661 & ,0667 \\
\hline 200 & 0327, & ,0399 & ,0442 & ,0473 & 0497, & ,0515 & 0531, & ,0543 & ,0554 & ,0564 & ,0572 & ,0579 & 0585, & ,0590 \\
\hline 250 & ,0262 & ,0320 & ,0356 & ,0381 & ,0400 & ,0416 & ,0429 & ,0439 & ,0449 & 0457, & ,0464 &, 0470 & 0475, & ,0480 \\
\hline $\bar{\chi}^{2}(\infty)$ & 6,635 & 8,144 & 9,092 & 9,784 & 10,327 & 10,774 & 11,152 & 11,480 & 11,768 & 12,025 & 12,258 & 12,469 & 12,662 & 12,842 \\
\hline
\end{tabular}


Tableau A2 $\backsim$ Valeurs critiques des tests $\bar{E}^{2}$ et $\bar{\chi}^{2}$ au seuil $\boldsymbol{\alpha}=\mathbf{0 , 0 1}$

\begin{tabular}{|c|c|c|c|c|c|c|c|c|c|c|c|c|c|c|}
\hline dle / $\mathrm{k}$ & 2 & 3 & 4 & 5 & 6 & 7 & 8 & 9 & 10 & 11 & 12 & 13 & 14 & 15 \\
\hline 2 & ,9604 & ,9531 & ,9289 & 8954 & ,8578 & 8195 & ,7822 & ,7469 & ,7138 & 6831 & ,6546 & ,6283 & ,6039 & ,5813 \\
\hline 3 & 8730, & 8781, & 8573, & 8278 & ,7951 & ,7621 & 7301, & 6996, & 6709, & 6440, & 6190, & 5957, & ,5740 & ,5538 \\
\hline 4 & 7783, & 7995, & ,7872 & ,7645 & ,7379 & 7103, & 6832 & 6570, & 6322 & 6087, & 5867, & ,5661 & ,5467 & ,5286 \\
\hline 5 & 6937, & 7276 & ,7236 & 7075, & 6866 & 6640, & 6411, & 6188, & 5973, & 5768, & 5575, & 5391, & 5219, & ,5056 \\
\hline 6 & 6221, & 6646, & 6674 & 6569 & 6409 & 6225, & 6034, & ,5844 & ,5658, & ,5479 & 5308, & ,5145, & 4991, & 4844 \\
\hline 7 & ,5622 & 6100 & 6181 & 6122 & 6003 & 5855, & ,5696 & ,5534 & 5373, & ,5216 & ,5065, & 4919, & 4781 & 4648 \\
\hline 8 & 5119, & ,5628 & ,5748 & ,5727 & ,5641 & 5523, & 5391 & ,5253, & 5113, & 4976, & 4842 & 4712, & 4587, & ,4467 \\
\hline 9 & 4694 & 5219, & 5368 & 5376, & 5317, & ,5225, & 5116, & 4998, & 4877, & 4756, & 4637, & 4521, & 4408, & ,4300 \\
\hline 10 & 4331, & 4862 & ,5032 & 5063, & ,5027 & 4956, & 4866 & 4765, & 4660 & 4554, & 4448, & 4344, & 4243, & ,4144 \\
\hline 11 & 4018, & 4549, & 4734 & 4783, & ,4765 & 4712, & 4638, & 4553, & 4462 & 4368, & 4274 & 4180, & 4089, & 3999 \\
\hline 12 & 3746, & 4272 & 4467, & 4531 & 4529 & 4490, & 4430, & 4358, & 4279, & 4196, & 4112, & 4028, & 3945, & 3863 \\
\hline 13 & 3508 & 4026 & 4229 & 4304 & 4314 & 4287, & 4239, & 4179, & 4110, & 4037, & ,3962 & 3886 & 3811 & ,3737 \\
\hline 14 & 3298 & ,3806 & 4013, & 4097 & 4118 & 4102, & 4064 & 4013, & 3954 & ,3890 & ,3822 & ,3754 & ,3686 & 3618 \\
\hline 15 & 3111, & 3608 & ,3818, & 3909 & 3938 & 3931, & 3902 & 3860 & 3809, & 3752, & ,3692 & 3631, & 3569 & ,3506 \\
\hline 16 & 2944, & ,3430 & 3641 & 3737, & 3773, & 3774 & 3753, & 3718, & ,3674 & 3624, & 3570 & 3515, & 3458, & 3401 \\
\hline 17 & 2793, & 3268 & 3479 & 3580 & ,3622 & 3629 & ,3614 & ,3586 & 3548, & ,3504 & ,3456 & ,3406 & 3354 & ,3302 \\
\hline 18 & 2657, & 3120 & 3331 & 3435 & 3481 & 3494 & ,3485 & ,3462 & 3430 & ,3392 & 3349 & ,3304 & ,3257 & ,3209 \\
\hline 19 & 2534 & 2985, & 3195, & 3301 & 3351, & 3369, & 3365 & 3347, & ,3320 & ,3286 & ,3248 & 3207, & 3164 & 3120 \\
\hline 20 & 2422 & 2861 & 3069 & 3177, & 3231 & 3252 & ,3253 & 3239, & ,3216 & 3187, & 3153, & ,3116 & 3077, & ,3037 \\
\hline 22 & 2224 & 2642 & 2844 & 2954 & 3013, & 3041, & ,3049 & 3043, & 3028 & ,3006 & 2979, & 2948, & 2916, & 2882 \\
\hline 24 & 2056, & 2453, & 2650 & 2761 & 2823 & 2856, & 2869 & 2869 & 2860 & 2843, & 2822 & 2798, & 2771 & ,2742 \\
\hline 26 & 1911, & 2289, & 2481, & 2591 & 2655, & 2691, & 2709, & 2714, & 2709, & 2698, & 2682 & 2662 & 2639, & 2615 \\
\hline 28 & 1786, & 2146 & 2332 & 2440 & 2506 & 2545, & 2566 & 2574, & 2573, & 2566, & 2554 & 2538, & 2520 & 2499 \\
\hline 30 & 1675, & 2020 & 2199 & 2306 & 2373 & 2413, & 2437, & 2448, & 2451, & 2447, & 2438, & 2426 & 2411 & 2393, \\
\hline 32 & 1578, & 1907, & 2081 & 2186 & 2253 & 2295, & 2320 & 2334 & 2339, & 2338 & 2332 & ,2323 & ,2310 & ,2296 \\
\hline 34 & 1491, & 1807, & 1975, & 2078, & 2144 & 2187, & 2214, & 2230 & 2237, & 2238, & 2235, & 2228 & ,2218 & ,2206 \\
\hline 36 & 1414, & 1716, & 1879, & 1980, & 2046 & 2089, & 2117, & 2134 & 2144 & 2147, & 2145, & 2140 & 2133 & 2123 \\
\hline 38 & 1344, & 1634, & 1792, & 1891, & 1956, & 1999, & 2028, & 2047, & 2057, & 2062 & 2063 & 2060 & 2054 & 2046 \\
\hline 40 & 1280, & 1560, & 1713, & 1809, & 1873, & 1917, & 1947, & 1966, & 1978, & 1984, & 1986, & 1985, & 1980, & 1974 \\
\hline 42 & 1222 & 1491, & 1640, & 1734, & 1798 & 1841, & 1871, & 1892, & 1905, & 1912, & 1915, & 1915, & 1912, & 1907, \\
\hline 44 & 1170, & 1429, & 1573, & 1665, & 1728 & 1771, & 1801, & 1822, & 1836, & 1845, & 1849, & 1850, & 1848, & 1845, \\
\hline 46 & 1121, & 1372, & 1512, & 1602, & 1663, & 1706, & 1737, & 1758, & 1773, & 1782, & 1787, & 1789, & 1789, & 1786, \\
\hline 48 & 1077, & 1319, & 1455, & 1543, & 1603, & 1646, & 1676, & 1698, & 1713, & 1723, & 1730, & 1733, & 1733, & 1731 \\
\hline 50 & 1036, & 1270, & 1402, & 1488, & 1547, & 1590, & 1620, & 1642, & 1658, & 1669, & 1675, & 1679, & 1680, & 1679, \\
\hline 55 & ,0945, & 1162 & 1285, & 1366, & 1423, & 1464, & 1495, & 1517, & 1534, & 1546, & 1554, & 1559, & 1562, & 1563, \\
\hline 60 & 0869, & 1071, & 1186, & 1263, & 1318, & 1358, & 1387, & 1410, & 1427, & 1439, & 1449, & 1455, & 1459, & 1461 \\
\hline 65 & 0805, & 0993, & 1102, & 1175, & 1227, & 1265, & 1294, & 1317, & 1334, & 1347, & 1357, & 1364, & 1369, & 1372, \\
\hline 70 & 0749, & ,0926, & 1028, & 1098, & 1147, & 1185, & 1213, & 1235, & 1252, & 1265, & 1276, & 1283, & 1289, & 1293, \\
\hline 75 & 0701, & 0867, & ,0964 & 1030, & 1078, & 1114, & 1141, & 1163, & 1180, & 1193, & 1204, & 1212, & 1218, & 1223, \\
\hline 80 & ,0658, & 0815, & ,0907 & 0970 & 1016, & 1051, & 1078, & 1099, & 1116, & 1129, & 1140, & 1148, & 1155, & 1160 \\
\hline 85 & ,0620 & ,0769 & 0857, & 0917, & ,0961 & 0994, & 1021, & 1041, & 1058, & 1071, & 1082, & 1091, & 1097, & 1103, \\
\hline 90 & 0587, & ,0728 & 0812 & 0869 & 0912 & ,0944 & 0969 & ,0990 & 1006, & 1019, & 1030, & 1039, & 1046, & 1051, \\
\hline 95 & 0557, & ,0691, & ,0771 & ,0826 & ,0867 & 0898, & 0923, & 0943, & 0959, & ,0972 & ,0982 & 0991, & 0998, & 1004 \\
\hline 100 & ,0529 & ,0658 & ,0735, & ,0787 & ,0827 & 0857, & 0881, & 0900, & ,0916, & 0929, & 0939, & 0948, & 0955, & ,0961 \\
\hline 125 & ,0425 & ,0530 & 0593 & 0637, & ,0670 & ,0696 & ,0717 & ,0734 & ,0748 & 0760 & ,0770 & 0778, & ,0786 & ,0792 \\
\hline 150 & ,0356, & ,0444 & ,0498 & 0535, & ,0564 & 0586 & ,0605 & ,0620 & ,0632 & 0643, & ,0652 & ,0660 & ,0667 & ,0673 \\
\hline 175 & ,0305, & ,0382 & ,0428 & ,0461 & ,0486 & ,0506, & 0523, & ,0536 & ,0548 & 0558, & ,0566 & 0573, & 0580 & ,0585 \\
\hline 200 & ,0268 & ,0335, & ,0376 & ,0405 & ,0428 & 0446, & ,0460 & 0473, & ,0483 & 0492, & ,0500 & 0507, & 0513, & ,0518 \\
\hline 250 & 0215, & ,0269 & ,0302 & ,0326 & ,0345 & ,0359 & ,0372 & ,0382 & 0391, & 0398, & ,0405 & 0411, & ,0416 & ,0421 \\
\hline $\bar{\chi}^{2}(\infty)$ & 5,412 & 6,823 & 7,709 & 8,356 & 8,865 & 9,284 & 9,638 & 9,946 & 10,216 & 10,457 & 10,676 & 10,875 & 11,056 & 11,225 \\
\hline
\end{tabular}


Tableau A3 - Valeurs critiques des tests $\bar{E}^{2}$ et $\bar{\chi}^{2}$ au seuil $\boldsymbol{\alpha}=\mathbf{0 , 0 2 5}$

\begin{tabular}{|c|c|c|c|c|c|c|c|c|c|c|c|c|c|c|}
\hline dle / $\mathrm{k}$ & 2 & 3 & 4 & 5 & 6 & 7 & 8 & 9 & 10 & 11 & 12 & 13 & 14 & 15 \\
\hline 2 & ,9025 & 8949 & 8603 & 8177 & ,7740 & ,7322 & ,6934 & 6578 & ,6253 & ,5958 & ,5688 & ,5442 & ,5216 & 5009 \\
\hline 3 & ,7715 & 7898 & ,7690 & 7378 & 7041 & 6709, & 6394 & 6101, & ,5829 & 5578 & ,5346 & ,5133 & 4935, & 4753, \\
\hline 4 & 6584, & 6971, & 6897, & 6690, & 6439, & 6180, & ,5925, & 5683, & ,5454 & ,5241 & 5041, & 4855, & 4682, & 4521, \\
\hline 5 & 5693, & 6201, & 6228, & 6104, & ,5922 & 5721, & ,5516 & 5315, & 5123, & 4941, & 4768, & 4606, & 4453, & 4310, \\
\hline 6 & 4995, & 5567, & ,5665, & ,5603, & ,5476 & 5321 & ,5156 & 4991, & 4828, & 4672, & ,4522 & 4380, & 4245, & 4117, \\
\hline 7 & 4441 & 5042 & ,5189 & ,5174 & 5089, & 4972, & 4839, & 4702, & 4564, & ,4430 & ,4300 & 4175, & 4055, & 3941, \\
\hline 8 & 3993, & 603 & 4783, & 4803, & 4751 & 4663, & 4557 & 4444, & 4327, & 4211, & 4097, & 3987, & ,3881, & 3779, \\
\hline 9 & 625 & 232 & 4433, & 4480, & 454 & 4390, & 4306, & 4211, & 4112, & 4012, & 3913, & 3816, & 3721, & 3629, \\
\hline 10 & 18 & 15 & 4130, & 96 & 0 & 46 & 0 & 4002, & 18 & 3831, & 14 & 3658, & 3573, & 3491, \\
\hline 11 & 3057 & 0 & ,3864 & 3945, & 6 & 3927, & & ,38 & & ,3666 & 89 & 3513, & 3437, & 3363, \\
\hline 12 & 2835, & 1 & 3630, & ,3722 & 45 & 729 & 1 & 3639, & 78 & ,3514 & 3447, & 3378, & 3311, & 3244, \\
\hline 13 & 2642 & 1 & ,3422 & 3523, & 56 & 51 & 23 & ,3480 & 3 & ,3373 & 14 & ,3254 & 3193, & 3132 \\
\hline 14 & 2473, & 5 & ,3237 & 3343, & 384 & 388 & 69 & 3335, & 3292 & ,3244 & ,3192 & 3138, & 3083, & 3029, \\
\hline 15 & 2325, & 2840 & 3070, & ,3181 & ,3228 & 239 & 3228, & 3201, & 3166, & ,3124 & 3078, & ,3030 & 2981, & 2931, \\
\hline 16 & 2193, & 691 & 2919, & 3033, & 3086 & 103 & 3098, & 3078, & 3048, & 3012, & 2972, & 2929, & 2885, & 2840, \\
\hline 17 & 775 & 557 & 2783, & 2898, & 2956 & 2978 & 2978, & 2963, & 2939, & 2908, & 2873, & 2835, & 2795, & 2754 \\
\hline 18 & 1969, & 2436, & 2658, & 2775, & 2836, & 2862 & 2867, & 2857, & 2838, & 2811, & 2781, & 2747, & 2711, & 2673, \\
\hline 19 & 1874, & 2326 & 2544 & 2662 & 2725, & 2755, & 2764, & 2758, & ,2743 & ,2720 & 2694 & 2663, & 2631, & 2597, \\
\hline 20 & 1787, & 225 & 2439, & 2557, & 2622 & 2655, & 2668, & 2666, & 2654, & 2635, & 2612, & 2585, & 2556, & 2525, \\
\hline 22 & 1635, & 047 & ,2254 & 2371, & 2439, & 2476, & 2494, & 2498, & 2492, & 2480, & 2462 & 2441, & 2418, & 2392 \\
\hline 24 & 07 & 395 & 2094, & 2210, & 2279, & 2320 & 2342, & 2350, & & 2342, & & 2313, & 2294 & 2273, \\
\hline 26 & 98 & & 6 & 2069, & 2, & 2 & & & & & 09 & & 21 & 2165, \\
\hline 28 & & & & & & & & & & & & & & \\
\hline 30 & & & & & & & & & & & & & & 1976, \\
\hline 32 & & & 2 & 1 & & & & & & & & & 1S & 1894, \\
\hline 34 & & & 1546, & 1 1, & & & & & & & & & 18 & 1818, \\
\hline 36 & 5 & & 1469, & 1 & & & & 1 & & & & & 17, & 1748, \\
\hline 38 & 73 & & 1400, & 14 & 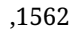 & 1 & , & 1661, & & & 38 & 1 & 16 & 1683, \\
\hline 40 & 0927, & & 1336, & 14 & & ) & & 1 & & & 24 & 16, & 1626, & 1623, \\
\hline 42 & 84 & & 1278 & 137, & & 8 & & 15, & & & & , 1 & 15, & 1567, \\
\hline 44 & 0845, & 1088, & 1225, & 1314, & 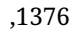 & 420 & 45 & 1475, & 1491, & 1503, & 1510, & 1514, & 1515, & 1515, \\
\hline 46 & 0810, & 14 & 1176, & 1263, & 32 & 7 & 35 & 1422, & 1 & 1 & 8 & 14, & 1466, & 1466, \\
\hline 48 & 0777, & 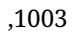 & 131 & 216 & 275 & 8 & 34 & 1373, & & 1402, & 0 & 14, & 1419, & 1420, \\
\hline 50 & ,0747 & 0965, & 08 & 172 & 230 & 2 & 30 & 1327, & 1 & 13 & 55 & 13, & 1375, & 1377, \\
\hline 55 & ,0681 & 0881, & 9997 & 075 & 130 & ) & 120 & 1224, & 1 & 1 & 5 & 1 & 1277, & 1280, \\
\hline 60 & 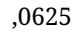 & , & 920 & ,0992 & & & & 1 & & & & & 1 & 1195, \\
\hline 65 & 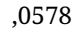 & , & 853 & ,0922 & , & & & 0 & & & & & & 1 \\
\hline 70 & & & & & & & & & & & & & & 1056, \\
\hline 75 & & & & & & & & & & & & & & 0998, \\
\hline 80 & & & & & & & & & & & & & & 09 \\
\hline 85 & & & 0662 & ,07 & & & & & & & & & & 0899 \\
\hline 90 & & 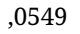 & ,06 & ,0680 & & & & & & & & & & ,0856 \\
\hline 95 & & תחת & 050 & ,06 & & & & & & & & &, $0 \xi$ & 0818, \\
\hline 100 & & & & 06 & & & & & & & & & 6 & ,0783 \\
\hline 125 & & & 456 & ,04 & 9528 & 2 & & & & & ,0622 & ,0630 & ,0637, & ,0644, \\
\hline 150 & ,0254 & 0334, & ,0382 & 04 & 0443 & 0464 & 04 & 0495, & ,0507 & 0518, & ,0526 & ,0534 & ,0541 & ,0546 \\
\hline 175 & ,0218 & ,0287 & 329 & 0359 & 2 & 1 & 04 & ,0428, & 39 & ,0448 & ,0456 & ,0463 & ,0469 & ,0475, \\
\hline 200 & 0191 & ,0251 & ,0289 & ,0315, & 0336 & ,0352 & ,0366 & 0377, & 0387, & ,0395, & ,0403 & ,0409 & ,0415, & ,0420 \\
\hline & 0152 & ,0202 & ,0232 & ,0254 & ,0270 & ,0284 & ,0295, & ,0304 & 0313, & ,0320 & ,0326 & ,0331, & ,0336 & 0341, \\
\hline $\bar{\chi}^{2}(\infty)$ & 3,841 & 5,098 & 5,891 & 6,471 & 6,928 & 7,304 & 7,624 & 7,901 & 8,145 & 8,363 & 8,561 & 8,740 & 8,905 & 9,058 \\
\hline
\end{tabular}


Tableau A4 $\backsim$ Valeurs critiques des tests $\bar{E}^{2}$ et $\bar{\chi}^{2}$ au seuil $\boldsymbol{\alpha}=\mathbf{0 , 0 5}$

\begin{tabular}{|c|c|c|c|c|c|c|c|c|c|c|c|c|c|c|}
\hline dle / $\mathrm{k}$ & 2 & 3 & 4 & 5 & 6 & 7 & 8 & 9 & 10 & 11 & 12 & 13 & 14 & 15 \\
\hline 2 & 8100 & 8111 & ,7730 & ,7278 & ,6834 & ,6424 & ,6053 & ,5719 & ,5419 & ,5149 & ,4905 & ,4684 & ,4482 & 4299 \\
\hline 3 & 6486 & 6866 & 6713, & 6430 & 6120 & 5814 & ,5527 & ,5261 & ,5017 & 4793, & 4588, & ,4400 & 4226 & 4066 \\
\hline 4 & 5319, & 5895, & 5900, & ,5742 & ,5530, & ,5304 & ,5082 & 4869, & 4669, & 4483, & 4308, & 4147, & 3997, & 3857, \\
\hline 5 & 4481, & ,5144 & 5249, & ,5178, & 5038, & 4873, & 4700, & 4529, & 4365, & 4208, & 4061, & 3921, & 3791 & 3668, \\
\hline 6 & 3862 & 4554 & 4721, & 4710, & 4623, & 4504, & 4370, & 4233, & 4097, & ,3966 & 3839, & 3719, & 3605, & 3496, \\
\hline 7 & 3390 & 1080 & 4287, & 4317, & 4270, & 4185, & 4082, & ,3972 & 3860 & ,3749 & ,3640 & 3535, & 3436 & 3340, \\
\hline 8 & 3018, & 694 & 3923, & ,3984 & 3966 & 3908, & 3830, & 3741, & 3648, & ,3554 & ,3461 & 3369, & ,3281 & 3196, \\
\hline 9 & 719 & 373 & 3615, & 3697, & 701 & 3665 & 3606, & 3535, & 3458, & 3378, & 3298, & 3218, & 3140, & 3065, \\
\hline 10 & 473 & 03 & 351 & 3448, & 99 & 449 & 407 & 50 & 3286, & 3219, & 3149, & 3080, & 3011, & 2943, \\
\hline 11 & 2267, & 2 & 3123, & ,3230 & 4 & 3258, & 3228, & ,3 & ,3 & 3073, & ,3014 & 2952 & 2892 & 2832 \\
\hline 12 & 2093, & 573 & 2923, & 3038, & (3t & 3086, & 3067, & 3032, & 2989, & 2941, & 2889, & 2835, & 2781, & 2728 \\
\hline 13 & 44 & 0 & 2748, & 2867, & 2918, & 2931, & 2921, & 2895, & 2860, & 2819, & 2774, & 2727, & 2679, & 2631, \\
\hline 14 & 14 & 7 & 2591, & 2714, & 2771, & 2791 & 2788, & 2769, & 2741, & 2707, & 2668, & 2627, & 2584, & 2541, \\
\hline 15 & 1700, & 2 & 2452, & 2576, & 2638, & 2664 & 2667, & 2654, & 2632, & 2603, & 2570, & 2534 & 2496, & 2457, \\
\hline 16 & 00 & 1 & 2327, & 2452, & 2518, & 2548, & 2555 & 2548, & 2531, & 2507, & 2478, & 2447, & 2413, & 2378, \\
\hline 17 & 11 & 883 & 2214, & 2339, & 24 & 2441, & 2453 & 2450, & 2437, & 2418, & 2393, & 2365, & 2336, & 2304 \\
\hline 18 & 1431, & 886 & 2111, & 2236 & 2306 & 2343, & 2358, & 2359, & 2350, & 2334, & 2314, & 2290 & 2263, & 2235, \\
\hline 19 & 1360, & 797 & 2017, & 2141, & 2213, & 2252 & 2271, & 2275, & 2269, & 2257, & 2239, & 2218, & 2195, & 2170, \\
\hline 20 & 1295, & 717 & 1932, & 2055, & 2127, & 2168, & 2189, & 2196, & 2194, & 2184, & 2169, & 2151, & 2130 & 2108, \\
\hline 22 & 1182, & 576 & 1780, & 1901, & 1974 & 2018, & 2043, & 2055, & 2057, & 2052, & 2042 & 2029, & 2013, & 1994, \\
\hline 24 & 87 & & 651 & 1768, & & 1887, & 1915 & 1930, & 1936, & 1935, & 1929, & 1919, & 1907, & 1893, \\
\hline 26 & 06 & & 9 & 2 & & & & & & & 28 & & & 1800, \\
\hline 28 & & & & & & & & & & & & & & , \\
\hline 30 & & & & & & & & & & & & & & 1641, \\
\hline 32 & 23 & & & & & & & & & & & 1 & & 1571, \\
\hline 34 & 6 & & ) & 0 & & & & & & & & 1 & & 1507, \\
\hline 36 & 4 & & 114, & 1 & & & & & & & 17 & 14, & 14, & 1448, \\
\hline 38 & ,0696 & 0 & 1 & 1 & & 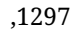 & 0 & 1 & & 1 & 89 & 1393, & 13 & 1394, \\
\hline 40 & ,0662 & 0905 & 3 & 113, & & & 1275 & 1 & 1 & 28 & 1336, & 13, & 13, & 1343, \\
\hline 42 & 1 & 4 & 09 & & & & 223 & 1 & & 1 & 1286, & 1292, & 1295, & 1296, \\
\hline 44 & ,0603 & 0827, & 0955, & 1040, & & 4 & 176 & 1200, & 1218, & 1231, & 1240, & 1247, & 1250, & 1252, \\
\hline 46 & 0577, & 0792, & 091, & ,09 & & 2 & 132 & 11, & 1 & 1 & 98 & 1204, & 1209, & 1211, \\
\hline 48 & ,0554 & ,076, & 88 & 096 & & ) & 2 & 11, & & 1 & 8 & 11, & 1170, & 1173, \\
\hline 50 & ,0532 & ,0732 & 848 & 926 & 982 & 023 & 05 & 10 & 1 & 1 & 20 & 1128, & 3 & 1137, \\
\hline 55 & ,0484 & 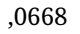 & 7775 & 0848, & 90 & .094 & 0970, & , $0 \varsigma$ & & 11, & 37 & 5 & 1 & 1055, \\
\hline 60 & 145 & , & ,0714 & 0783, & , & 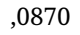 & 089 & & & 0 & 55 & & 0 & 0985, \\
\hline 65 & , & & 662 & 726 & 0 & 9 & & & & , & & & 8 & ,0924 \\
\hline 70 & & & & & & & & & & & & & & 0869 \\
\hline 75 & & & & & & & & & & & & & & 0821 \\
\hline 80 & & & & & & & & & & & & & & ,0778 \\
\hline 85 & & & ,05 & & & & & & & & & & & ,0739 \\
\hline 90 & & & 0485 & & & & & & & & & & & ,0704 \\
\hline 95 & & 02 & ,0460 & & & & & & & & & & & ,0672 \\
\hline 100 & & & & & & & & & & & ,0620 & & & ,0643 \\
\hline 125 & & & 25 & & & & & & & ,0497 & ,0507 & 0515 & ,0522 & ,0528 \\
\hline 150 & 0179 & 0251, & 0295, & ,0327 & בר & 370 & 0386, & ,03 & ,0410 & ,0420 & ,0428 & ,0436 & ,0442 & 0448, \\
\hline 175 & 0154 & ,0216 & 254 & ,0281 & 30 & 319 & 0333, & 03 & 03 & ,0364 & ,0371 & ,0378 & ,0384 & ,0389 \\
\hline 200 & ,0135 & 0189, & 0223, & ,0247 & 0266 & 0281 & ,0293 & ,0304 & 0313, & ,0321 & ,0327 & ,0333 & ,0339 & ,0344 \\
\hline & ,0108 & 015 & ,017, & 0198, & ,0214 & ,0226 & ,0236 & ,0245, & ,0252 & ,0259 & ,0265 & ,0270 & ,0275, & 0279, \\
\hline $\bar{\chi}^{2}(\infty)$ & 2,706 & 3,820 & 4,528 & 5,049 & 5,460 & 5,800 & 6,088 & 6,339 & 6,560 & 6,758 & 6,937 & 7,100 & 7,250 & 7,389 \\
\hline
\end{tabular}


Listing du programme de détermination des coefficients du contraste optimal pour l'étude d'une série de $k$ éléments soumis au test de variation monotone selon Barlow et coll. (1972)

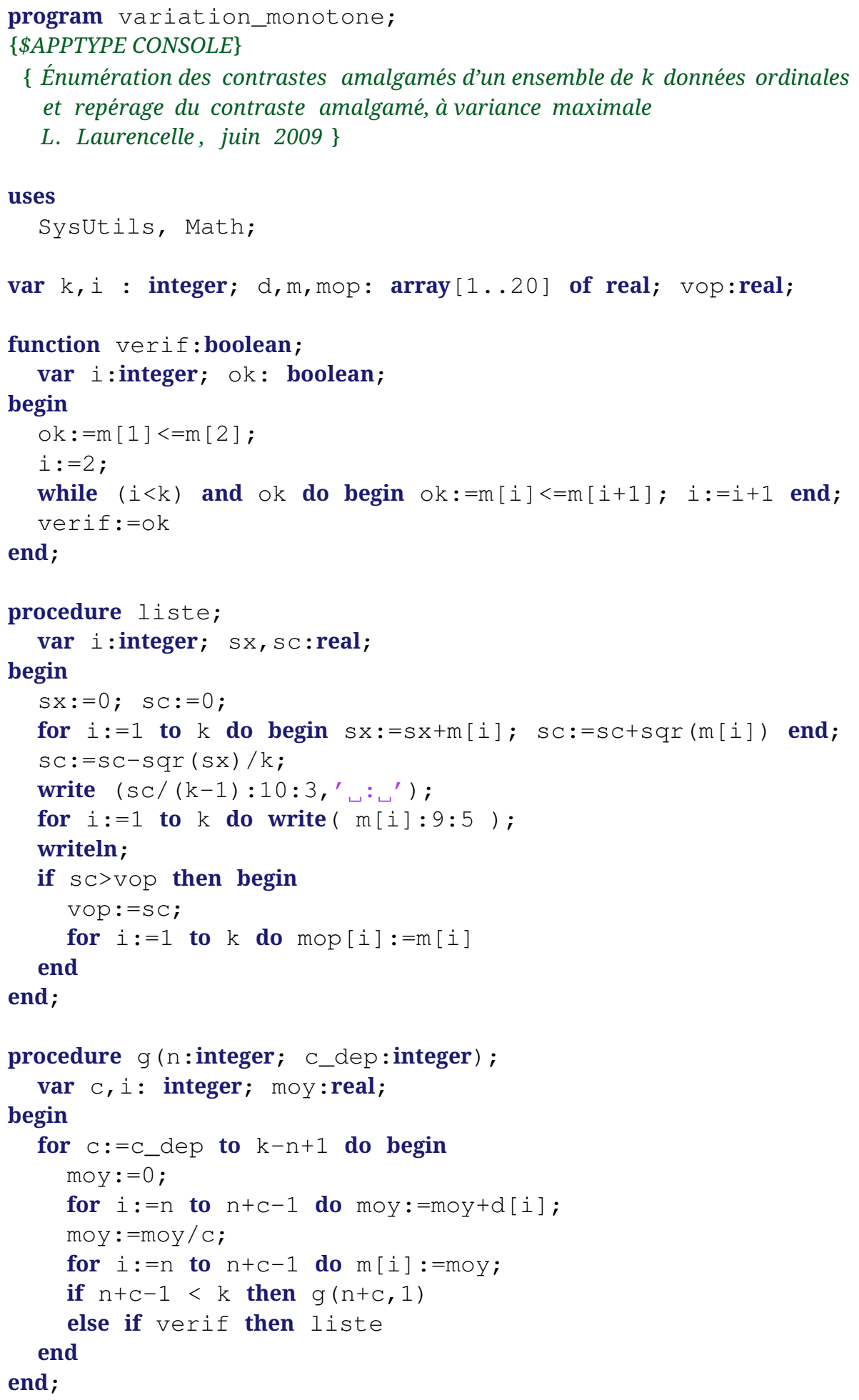




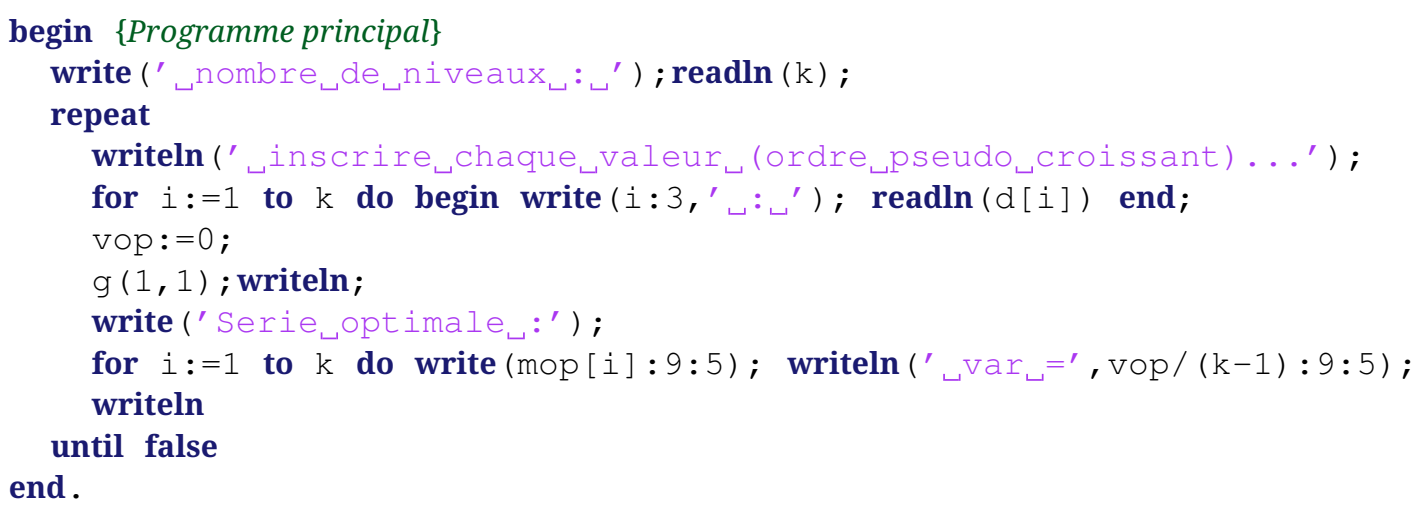

\section{Coefficients $c_{j}$ du contraste linéaire selon Abelson et Tukey (2014), incluant la somme de leurs valeurs carrées*}

\begin{tabular}{|c|c|c|c|c|c|c|c|c|c|c|c|c|c|c|}
\hline 4 & $-0,866$ & $-0,134$ & $(1,536)$ & & & & & & & & & & & \\
\hline 5 & $-0,894$ & $-0,201$ & 0,000 & $(1,679)$ & & & & & & & & & & \\
\hline 6 & $-0,913$ & $-0,242$ & $-0,070$ & $(1,794)$ & & & & & & & & & & \\
\hline 7 & $-0,926$ & $-0,269$ & $-0,114$ & 0,000 & $(1,886)$ & & & & & & & & & \\
\hline 8 & $-0,935$ & $-0,289$ & $-0,145$ & $-0,045$ & $(1,962)$ & & & & & & & & & \\
\hline 9 & $-0,943$ & $-0,304$ & $-0,167$ & $-0,076$ & 0,000 & $(2,031)$ & & & & & & & & \\
\hline 10 & $-0,949$ & $-0,316$ & $-0,184$ & $-0,100$ & $-0,032$ & $(2,091)$ & & & & & & & & \\
\hline 11 & $-0,953$ & $-0,326$ & $-0,198$ & $-0,118$ & $-0,056$ & 0,000 & $(2,141)$ & & & & & & & \\
\hline 12 & $-0,957$ & $-0,334$ & $-0,209$ & $-0,133$ & $-0,075$ & $-0,024$ & $(2,190)$ & & & & & & & \\
\hline 13 & $-0,961$ & $-0,340$ & $-0,218$ & $-0,145$ & $-0,090$ & $-0,043$ & 0,000 & $(2,235)$ & & & & & & \\
\hline 14 & $-0,964$ & $-0,346$ & $-0,226$ & $-0,155$ & $-0,103$ & $-0,059$ & $-0,019$ & $(2,277)$ & & & & & & \\
\hline 15 & $-0,966$ & $-0,350$ & $-0,233$ & $-0,164$ & $-0,113$ & $-0,072$ & $-0,035$ & 0,000 & $(2,312)$ & & & & & \\
\hline 16 & $-0,968$ & $-0,355$ & $-0,238$ & $-0,171$ & $-0,122$ & $-0,082$ & $-0,048$ & $-0,016$ & $(2,346)$ & & & & & \\
\hline 17 & $-0,970$ & $-0,358$ & $-0,243$ & $-0,177$ & $-0,130$ & $-0,092$ & $-0,059$ & $-0,029$ & 0,000 & $(2,378)$ & & & & \\
\hline 18 & $-0,972$ & $-0,362$ & $-0,248$ & $-0,183$ & $-0,136$ & $-0,100$ & $-0,068$ & $-0,040$ & $-0,013$ & $(2,411)$ & & & & \\
\hline 19 & $-0,973$ & $-0,364$ & $-0,252$ & $-0,188$ & $-0,142$ & $-0,107$ & $-0,076$ & $-0,049$ & $-0,024$ & 0,000 & $(2,437)$ & & & \\
\hline 20 & $-0,975$ & $-0,367$ & $-0,255$ & $-0,192$ & $-0,148$ & $-0,113$ & $-0,084$ & $-0,058$ & $-0,034$ & $-0,011$ & $(2,467)$ & & & \\
\hline 21 & $-0,976$ & $-0,369$ & $-0,258$ & $-0,196$ & $-0,152$ & $-0,118$ & $-0,090$ & $-0,065$ & $-0,042$ & $-0,021$ & 0,000 & $(2,491)$ & & \\
\hline 22 & $-0,977$ & $-0,371$ & $-0,261$ & $-0,199$ & $-0,157$ & $-0,123$ & $-0,096$ & $-0,072$ & $-0,050$ & $-0,029$ & $-0,010$ & $(2,515)$ & & \\
\hline 23 & $-0,978$ & $-0,373$ & $-0,264$ & $-0,203$ & $-0,160$ & $-0,128$ & $-0,101$ & $-0,077$ & $-0,056$ & $-0,037$ & $-0,018$ & 0,000 & $(2,539)$ & \\
\hline 24 & $-0,979$ & $-0,375$ & $-0,266$ & $-0,206$ & $-0,164$ & $-0,132$ & $-0,105$ & $-0,083$ & $-0,062$ & $-0,044$ & $-0,026$ & $-0,009$ & $(2,562)$ & \\
\hline 25 & $-0,980$ & $-0,377$ & $-0,268$ & $-0,208$ & $-0,167$ & $-0,135$ & $-0,110$ & $-0,087$ & $-0,068$ & $-0,049$ & $-0,032$ & $-0,016$ & 0,000 & $(2,583)$ \\
\hline
\end{tabular}

* Les coefficients présentés réfèrent à une série qui serait croissante, les signes pouvant être inversés en vue d’une hypothèse de décroissance; cependant le calcul final étant mis au carré, la polarité du contraste n’influence pas le test. Seule la première moitié des coefficients est affichée, l'autre moitié étant formée en miroir, les valeurs voyant leur signe s'inverser. Quant à la quantité donnée en parenthèse, elle correspond ici à $2 \times \sum c_{j}^{2}$ avec les coefficients présentés. Par exemple, pour $k=5, \sum c_{j}^{2}=2 \times\left[\left(-0,894^{2}\right)+\left(-0.201^{2}\right)+\left(0,000^{2}\right]=2 \times 0,839637=1,679274 \approx 1,679\right.$.

\section{Citation}

LAURENCELlE, L. (2021d). L'analyse de variation monotone pour les moyennes et les proportions. The Quantitative Methods for Psychology, 17(4), 374-390. doi :10.20982/tqmp.17.4.p374

Copyright (C) 2021, LAURENCELLE. This is an open-access article distributed under the terms of the Creative Commons Attribution License (CC BY). The use, distribution or reproduction in other forums is permitted, provided the original author(s) or licensor are credited and that the original publication in this journal is cited, in accordance with accepted academic practice. No use, distribution or reproduction is permitted which does not comply with these terms.

Received: $12 / 10 / 2021$ 